\title{
Star product realizations of $\kappa$-Minkowski space
}

\author{
Bergfinnur Durhuus and Andrzej Sitarz
}

\begin{abstract}
We define a family of star products and involutions associated with $\kappa$-Minkowski space. Applying corresponding quantization maps we show that these star products restricted to a certain space of Schwartz functions have isomorphic Banach algebra completions. For two particular star products it is demonstrated that they can be extended to a class of polynomially bounded smooth functions allowing a realization of the full Hopf algebra structure on $\kappa$ Minkowski space. Furthermore, we give an explicit realization of the action of the $\kappa$-Poincaré algebra as an involutive Hopf algebra on this representation of $\kappa$-Minkowski space and initiate a study of its properties.
\end{abstract}

Mathematics Subject Classification (2010). 46L65, 53D55, 81R60.

Keywords. Quantization, deformation, star product, $\kappa$-Minkowski space, quantum Poincaré algebra.

\section{Contents}

1 Introduction . . . . . . . . . . . . . . . . . . . 606

2 Quantizations and star products . . . . . . . . . . . . . . . . 609

2.1 The right-invariant case . . . . . . . . . . . . . . . . . . . . . 609

2.2 The left-invariant case and other star products . . . . . . . . . . . . . 616

3 The extended algebra . . . . . . . . . . . . . . . . . . . . . 619

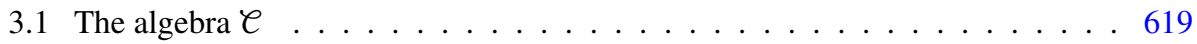

3.2 Hopf algebra properties . . . . . . . . . . . . . . . . . . . 6 . . . . . . . . . . . . 624

4 Lorentz covariance . . . . . . . . . . . . . . . . . . . . 630

4.1 Action of the momentum subalgebra on $€ \ldots \ldots 631$

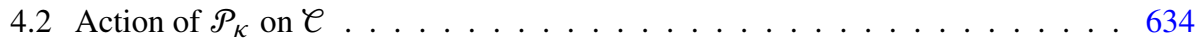

4.3 Explicit dependence on the kappa parameter . . . . . . . . . . . . . . 639

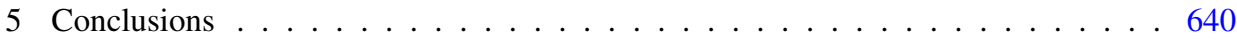

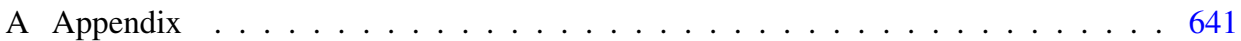

References . . . . . . . . . . . . . . . . . . . . . . . 644 


\section{Introduction}

The $\kappa$-deformation of Minkowski space was originally proposed in [11] as a Hopf algebra whose underlying algebra is the enveloping algebra of the Lie algebra with $d$ generators $x_{0}, \ldots, x_{d-1}$ fulfilling

$$
\left[x_{0}, x_{i}\right]=\frac{i}{\kappa} x_{i}, \quad\left[x_{i}, x_{j}\right]=0, \quad i, j=1, \ldots, d-1,
$$

where $\kappa \neq 0$ can be viewed as a deformation parameter since, formally, in the limit $\kappa \rightarrow \infty$ one obtains the commutative coordinate algebra of Minkowski space. Of course, to single out this limit as the Minkowski space requires some additional structure involving the action of the Poincaré group, or rather a deformed version thereof, for finite $\kappa[10]$. This was how the algebra originally was conceived and we shall return to this issue in Section 4. For the moment we concentrate on (1).

The first object of this paper is to discuss a class of star products on $\mathbb{R}^{d}$ and associated quantization maps based on the harmonic analysis on the Lie group associated with (1). The motivation originates from a similar approach to the standard Weyl quantization map based on its relation to the Heisenberg algebra of quantum mechanics. For the purpose of later reference let us briefly recall the main steps in this construction. The Heisenberg algebra associated to a particle moving on the real line is the three-dimensional Lie algebra defined by the relation

$$
[P, Q]=i C,
$$

where $C$ is a central element. The real form of this algebra with basis $i P, i Q, i C$ has a faithful representation $\sigma$ in terms of strictly upper triangular matrices:

$$
\sigma(i(a P+b Q+c C))=\left(\begin{array}{ccc}
0 & a & c \\
0 & 0 & b \\
0 & 0 & 0
\end{array}\right) .
$$

The connected and simply connected Lie group of the algebra is by definition the Heisenberg group, which we denote by Heis. It is the group of upper triangular matrices of the form

$$
T(a, b, c)=\left(\begin{array}{ccc}
1 & a & c+\frac{1}{2} a b \\
0 & 1 & b \\
0 & 0 & 1
\end{array}\right),
$$

which is obtained by exponentiation of (2). The group operations, expressed in this parametrization, are seen to be

$$
\begin{aligned}
T(a, b, c) T\left(a^{\prime}, b^{\prime}, c^{\prime}\right) & =T\left(a+a^{\prime}, b+b^{\prime}, c+c^{\prime}+\frac{1}{2}\left(a b^{\prime}-a^{\prime} b\right)\right), \\
T(a, b, c)^{-1} & =T(-a,-b,-c) .
\end{aligned}
$$

It follows that $\mathscr{H}$ eis is a unimodular group with Haar measure equal to $d a d b d c$. Thus the convolution algebra of integrable functions on $\mathscr{H}$ eis can be identified with $\mathrm{L}^{1}\left(\mathbb{R}^{3}\right)$ 
via the parametrization (3). Let $\circ$ denote the corresponding convolution product on $\mathrm{L}^{1}\left(\mathbb{R}^{3}\right)$.

According to the Stone-von Neumann theorem [15] the non-trivial irreducible unitary representations of $\mathscr{H e i s}$ are labeled by the value $\hbar \neq 0$ of the central element $C$. Fixing $\hbar$, the representation $\pi$ can be expressed in the form

$$
\begin{aligned}
\pi(T(a, b, c)) & =e^{\frac{i}{2} \hbar c} U(a, b), \\
(U(a, b) \psi)(x) & =e^{\frac{i}{2} \hbar a b} e^{i b x} \psi(x-\hbar a)
\end{aligned}
$$

for $\psi \in \mathrm{L}^{2}(\mathbb{R})$. The corresponding representation of the convolution algebra, also denoted by $\pi$, is then given by

$$
\pi(F)=\int_{\mathbb{R}^{3}} d a d b d c F(a, b, c) \pi(T(a, b, c))=\int_{\mathbb{R}^{2}} d a d b F^{\sharp}(a, b) U(a, b),
$$

where

$$
F^{\sharp}(a, b)=\int d c F(a, b, c) e^{-i \hbar c} .
$$

Clearly, $F \rightarrow F^{\sharp}$ maps $\mathrm{L}^{1}\left(\mathbb{R}^{3}\right)$ onto $\mathrm{L}^{1}\left(\mathbb{R}^{2}\right)$ and a simple calculation yields

$$
(F \circ G)^{\sharp}(a, b)=\int_{\mathbb{R}^{2}} d a^{\prime} d b^{\prime} F^{\sharp}(a, b) G^{\sharp}\left(a-a^{\prime}, b-b^{\prime}\right) e^{\frac{i}{2}\left(a b^{\prime}-a^{\prime} b\right)},
$$

where the last expression is a "twisted" convolution product on $\mathbb{R}^{2}$ that we shall denote by $F^{\sharp} \hat{\circ} G^{\sharp}$, and where we have set $\hbar=1$ for the sake of simplicity.

According to (5) we may write $\pi(f)$ instead of $\pi(F)$ when $f=F^{\sharp}$. With this notation the Weyl quantization map $W$ is defined by

$$
W(f)=\pi(\mathcal{F} f)
$$

for $f \in \mathrm{L}^{1}\left(\mathbb{R}^{2}\right) \cap \mathcal{F}^{-1}\left(\mathrm{~L}^{1}\left(\mathbb{R}^{2}\right)\right)$, where $\mathcal{F}$ denotes the Fourier transform on $\mathbb{R}^{2}$,

$$
(\mathcal{F} f)(a, b)=\frac{1}{2 \pi} \int d \alpha d \beta f(\alpha, \beta) e^{-i(a \alpha+b \beta)} .
$$

Using

$$
\pi(F \circ G)=\pi(F) \pi(G) \text { for } F, G \in \mathrm{L}^{1}\left(\mathbb{R}^{3}\right),
$$

we obtain from (5) and (6) that

$$
W\left(f *_{0} g\right)=W(f) W(g),
$$

where $f$ and $g$ are e.g. Schwartz functions on $\mathbb{R}^{2}$ and their Weyl product is defined by

$$
f *_{0} g=\mathscr{F}^{-1}((\mathcal{F} f) \hat{\circ}(\mathcal{F} g)) \text {. }
$$


From this definition the familiar expressions (see e.g. [7]) for the Weyl product can easily be derived. Likewise, the Weyl operators $W(f)$ can be seen to be integral operators for appropriate functions $f$. In particular, it can be shown that $W(f)$ is of Hilbert-Schmidt type if and only if $f$ is square integrable, and in this case

$$
\|W(f)\|_{2}^{2}=2 \pi \int d \alpha d \beta|f(\alpha, \beta)|^{2},
$$

where $\|\cdot\|_{2}$ denotes the Hilbert-Schmidt norm. It follows that the Weyl product can be extended to square integrable functions and $W$ can be extended to an isomorphism between the resulting algebra and the Hilbert-Schmidt operators on $\mathrm{L}^{2}(\mathbb{R})$.

It is worth emphasizing that the construction just outlined depends on the chosen parametrization of the Heisenberg group. An alternative parametrization preserving the invariant measure is, e.g.,

$$
(a, b, c) \rightarrow T(a, b, c+\xi a b)
$$

where $\xi$ is a real constant. In this case, one obtains a quantization map $W_{\xi}$ and a star product $*_{\xi}$ that are related to $*_{0}$ by

$$
W_{\xi}(f)=W\left(\Psi_{\xi} f\right), \quad \Psi_{\xi}\left(f *_{\xi} g\right)=\left(\Psi_{\xi} f\right) *_{0}\left(\Psi_{\xi} g\right),
$$

where $\Psi_{\xi}$ is defined by

$$
\left(\Psi_{\xi} f\right)(\alpha, \beta)=e^{i \xi \alpha \beta} f(\alpha, \beta)
$$

It follows that $\Psi_{\xi}$ is an isomorphism of star algebras of Schwartz functions and, moreover, since both $\mathcal{F}$ and multiplication by a phase factor preserve the norm in $\mathrm{L}^{2}\left(\mathbb{R}^{2}\right)$, we have that (8) is also fulfilled with $W$ replaced by $W_{\xi}$. In particular, one can verify that $W_{-\frac{1}{2}}$ is the so-called Kohn-Nirenberg quantization map, in which case $W_{-\frac{1}{2}}(f)$ is the pseudo-differential operator with symbol $f$. The Weyl map is singled out among the maps $W_{\xi}$ by the property

$$
W(f)^{*}=W(\bar{f})
$$

where $\bar{f}$ is the complex conjugate of $f$.

The purpose of this paper is to develop an approach similar to the preceding to quantization maps associated with $\kappa$-Minkowski space for $d=2$, which we denote by $M_{\kappa}$. In Section 2 we introduce the $\kappa$-Minkowski group $G$, analogous to Heis, and via harmonic analysis on $G$ we define a family of products, called star products, and involutions for a class $\mathscr{B}$ of Schwartz functions on $\mathbb{R}^{2}$. Explicit expressions for the star products and operator kernels are obtained which are used to show that those involutive algebras have natural isomorphic Banach algebra completions. In Section 3 two particular star products associated to the left and right-invariant Haar measures on $G$ are discussed. It is shown that they have natural extensions to a 
certain subalgebra $\mathcal{C}$ of the multiplier algebra of $\mathscr{B}$ consisting of smooth functions of polynomial growth. Moreover, it is shown that the resulting algebra has a Hopf star algebra structure furnishing a star product representation of $\kappa$-Minkowski space. In Section 4 we show how to represent the action of the $\kappa$-Poincaré algebra $\mathcal{P}_{\kappa}$ on $\kappa$ Minkowski space in this particular realization as well as on the subalgebra $\mathcal{B}$. On the latter we show that the Lebesgue integral is a twisted trace, invariant under the action of $\mathcal{P}_{\kappa}$. Finally, Section 5 contains some concluding remarks and a few technical details are collected in an appendix.

\section{Quantizations and star products}

2.1. The right-invariant case. In the following we restrict attention to $d=2$ in which case the Lie algebra defined by (1) is the unique noncommutative Lie algebra of dimension 2 and $\kappa$-Minkowski space $M_{\kappa}$ is its universal enveloping algebra. We set $x=x_{1}$ and $t=\kappa x_{0}$ and consider the real form of the Lie algebra with generators $i t, i x$ fulfilling

$$
[t, x]=i x .
$$

It has a faithful 2-dimensional representation $\rho$ given by

$$
\rho(i t)=\left(\begin{array}{cc}
-1 & 0 \\
0 & 0
\end{array}\right), \quad \rho(i x)=\left(\begin{array}{ll}
0 & 1 \\
0 & 0
\end{array}\right),
$$

and the corresponding connected and simply connected Lie group is the group $G$ of $(2 \times 2)$-matrices of the form

$$
S(a, b)=\left(\begin{array}{cc}
e^{-a} & b \\
0 & 1
\end{array}\right), \quad a, b \in \mathbb{R},
$$

obtained by exponentiating $\rho$ :

$$
e^{i \rho\left(a t+b^{\prime} x\right)}=\left(\begin{array}{cc}
e^{-a} & \frac{1-e^{-a}}{a} b^{\prime} \\
0 & 1
\end{array}\right) .
$$

The group operations written in the $(a, b)$ coordinates become

$$
S\left(a_{1}, b_{1}\right) S\left(a_{2}, b_{2}\right)=S\left(a_{1}+a_{2}, b_{1}+e^{-a_{1}} b_{2}\right), \quad S(a, b)^{-1}=S\left(-a,-e^{a} b\right) .
$$

An immediate consequence is

Lemma 2.1. The Lebesgue measure $d a d b$ is right-invariant, whereas the measure $e^{a} d a d b$ is left-invariant on $G$. In particular, $G$ is not unimodular.

Let $\mathcal{A}$ denote the convolution algebra of integrable functions on $G$ with respect to the right-invariant measure. Identifying functions on $G$ with functions on $\mathbb{R}^{2}$ by the 
parametrization (10), $\mathcal{A}$ is then the involutive Banach algebra consisting of integrable functions on $\mathbb{R}^{2}$ with product $\hat{*}$ and involution ${ }^{\dagger}$ given by

$$
\begin{aligned}
(f \hat{*} g)(a, b) & =\int d a^{\prime} d b^{\prime} f\left(a-a^{\prime}, b-e^{a^{\prime}-a} b^{\prime}\right) g\left(a^{\prime}, b^{\prime}\right), \\
f^{\dagger}(a, b) & =e^{a} \bar{f}\left(-a,-e^{a} b\right),
\end{aligned}
$$

where $f, g \in \mathcal{A}$ and $\bar{f}$ is the complex conjugate of $f$. If $\pi$ is a unitary representation of $G$ (always assumed to be strongly continuous in what follows) it is well known (see e.g. [16]) that $\pi$ defines a representation of $\mathcal{A}$ (which we shall also denote by $\pi$ ) by setting

$$
\pi(f)=\int d a d b f(a, b) \pi(S(a, b)) .
$$

Thus, we have

$$
\pi(f \hat{*} g)=\pi(f) \pi(g) \text { and } \pi\left(f^{\dagger}\right)=\pi(f)^{*} .
$$

Following the same procedure as described for the Weyl quantization above we define the Weyl map $W_{\pi}$ associated with the representation $\pi$ by

$$
W_{\pi}(f)=\pi(\mathcal{F} f) \text { for } f \in \mathrm{L}^{1}\left(\mathbb{R}^{2}\right) \cap \mathcal{F}^{-1}\left(\mathrm{~L}^{1}\left(\mathbb{R}^{2}\right)\right),
$$

where $\mathcal{F}$ denotes the Fourier transform (7) on $\mathbb{R}^{2}$. It then follows from (14) that

$$
W_{\pi}(f * g)=W_{\pi}(f) W_{\pi}(g) \quad \text { and } \quad W_{\pi}\left(f^{*}\right)=W_{\pi}(f)^{*}
$$

where the $*$-product and the ${ }^{*}$-involution are defined by

$$
f * g=\frac{1}{2 \pi} \mathscr{F}^{-1}((\mathcal{F} f) \hat{*}(\mathscr{F} g)) .
$$

and

$$
f^{*}=\mathscr{F}^{-1}\left(\mathcal{F}(f)^{\dagger}\right),
$$

respectively. As in the case of the standard Moyal product, one needs to exercise care about the domain of definition for the right-hand sides of (15) and (16). In this section we restrict our attention to the subset $\mathcal{B}$ of Schwartz functions introduced in the following definition, while an extension to a class of polynomially bounded functions will be discussed in subsequent sections.

Definition 2.2. Let $S_{\mathrm{c}}$ denote the space of Schwartz functions on $\mathbb{R}^{2}$ with compact support in the first variable, i.e., $\operatorname{supp}(f) \subseteq K \times \mathbb{R}$, where $K \subseteq \mathbb{R}$ is compact. Then we define $\mathscr{B}=\mathscr{F}\left(S_{\mathrm{c}}\right)=\mathscr{F}^{-1}\left(S_{\mathrm{c}}\right)$. 
Proposition 2.3. If $f, g \in \mathcal{B}$, then $f^{*}$ and $f * g$ also belong to $\mathcal{B}$ and are given by

$$
f * g(\alpha, \beta)=\frac{1}{2 \pi} \int d v \int d \alpha^{\prime} f\left(\alpha+\alpha^{\prime}, \beta\right) g\left(\alpha, e^{-v} \beta\right) e^{-i \alpha^{\prime} v},
$$

and

$$
f^{*}(\alpha, \beta)=\frac{1}{2 \pi} \int d v \int d \alpha^{\prime} \bar{f}\left(\alpha+\alpha^{\prime}, e^{-v} \beta\right) e^{-i \alpha^{\prime} v},
$$

respectively.

Proof. Invariance of $\mathcal{F}^{-1}\left(S_{\mathrm{c}}\right)$ under the $*$-product and the ${ }^{*}$-involution follows from the fact that $S_{\mathrm{c}}$ is an involutive subalgebra of the convolution algebra $\mathcal{A}$ as is easily seen from (12) and (13).

In order to establish (17), note that its right-hand side equals

$$
\frac{1}{\sqrt{2 \pi}} \int d v \tilde{f}(v, \beta) g\left(\alpha, e^{-v} \beta\right) e^{i \alpha v},
$$

where $\tilde{f}$ denotes the Fourier transform of $f$ with respect to the first variable

$$
\tilde{f}(a, \beta)=\frac{1}{\sqrt{2 \pi}} \int d \alpha f(\alpha, \beta) e^{-i a \alpha} .
$$

Note also that the integrand in (19) is a Schwartz function of $v, \alpha, \beta$ with compact support in $v$. Thus it suffices to show that the Fourier transform of (19) with respect to $\alpha, \beta$ equals $(2 \pi)^{-1} \mathcal{F} f \hat{*} \mathcal{F} g$. This follows from a straightforward calculation using the Plancherel theorem on the $\beta$-integral.

Concerning (18) we note similarly that the right-hand side equals

$$
\frac{1}{\sqrt{2 \pi}} \int d v \overline{\tilde{f}}\left(-v, e^{-v} \beta\right) e^{i \alpha v} .
$$

It is seen that the integrand is a Schwartz function of $v, \beta$ and that Fourier transforming (20) with respect to $\beta$ gives

$$
\frac{1}{\sqrt{2 \pi}} \int d v \overline{\mathcal{F} f}\left(-v,-e^{v} b\right) e^{v} e^{i \alpha v}=\frac{1}{\sqrt{2 \pi}} \int d v(\mathcal{F} f)^{\dagger}(v, b) e^{i \alpha v} .
$$

Hence, by Fourier inversion and (16) we conclude that the Fourier transform of the right-hand side of (18) equals $\mathcal{F}\left(f^{*}\right)$. This proves (18).

Note that the associativity of the above defined star product on $\mathcal{B}$ is an immediate consequence of associativity of the convolution product on $\mathcal{A}$. Likewise, $f \rightarrow f^{*}$ is an involution on $\mathcal{B}$, since $f \rightarrow f^{\dagger}$ is an involution on $\mathcal{A}$. Thus we have

Corollary 2.4. $B$ equipped with the *-product and ${ }^{*}$-involution defined by (17) and (18) is an involutive algebra. 
It should be noted that the star product and the involution as defined by (15) and (16) are independent of the choice of representation $\pi$ of $G$, while the quantization map $W_{\pi}$ that we proceed to discuss next is indeed representation dependent. As $G$ is isomorphic to the identity component of the group of affine transformations on $\mathbb{R}$, its representation theory is well known [8]. In particular, there is a close relationship to the representation theory of the Heisenberg group [1]. We recall the basic result, which we shall use later, and the proof of which we include for the sake of completeness (see also [2]).

Proposition 2.5. G has exactly two non-trivial irreducible unitary representations $\pi_{ \pm}$. Their action on the generators $t, x$ is given by

$$
\begin{aligned}
& \pi_{+}(t)=-i \frac{d}{d s}, \quad \pi_{+}(x)=e^{-s}, \\
& \pi_{-}(t)=-i \frac{d}{d s}, \quad \pi_{-}(x)=-e^{-s},
\end{aligned}
$$

as self-adjoint operators on $\mathrm{L}^{2}(\mathbb{R})$.

All other irreducible unitary representations are one-dimensional of the form $\pi_{c}(x)=0$ and $\pi_{c}(t)=c$ for some $c \in \mathbb{R}$.

Proof. Let $\pi$ be a unitary representation of $G$ on a Hilbert space $H$ and let $v \in$ $\operatorname{dom}(\pi(x))$. Differentiating the relation

$$
e^{i a \pi(t)} e^{i b \pi(x)} e^{-i a \pi(t)} v=e^{i b e^{-a} \pi(x)} v,
$$

which follows from (11), with respect to $b$ we get $e^{-i a \pi(t)} v \in \operatorname{dom}(\pi(x))$ and

$$
e^{i a \pi(t)} \pi(x) e^{-i a \pi(t)} v=e^{-a} \pi(x) v,
$$

so the two self-adjoint operators $e^{i a \pi(t)} \pi(x) e^{-i a \pi(t)}$ and $e^{-a} \pi(x)$ coincide. Since $e^{-a}>0$, the spectral subspaces $H_{+}, H_{-}$and $H_{0}$ corresponding to the positive and negative real line and $\{0\}$, respectively, are identical for $\pi(x)$ and $e^{i a \pi(t)} \pi(x) e^{-i a \pi(t)}$. It follows that those spaces are invariant under $e^{i a \pi(t)}$ and $e^{i b \pi(x)}$. By irreducibility, one of them equals $H$ and the other two vanish.

Assume that $H=H_{+}$and define the self-adjoint operator $Q$ by

$$
Q=-\ln (\pi(x))
$$

Then $\pi(x)=e^{-Q}$ and by (23) we have

$$
\exp \left(i b e^{i a \pi(t)} e^{-Q} e^{-i a \pi(t)}\right)=\exp \left(i b e^{-a} e^{-Q}\right),
$$

and hence

$$
e^{i a \pi(t)} e^{-Q} e^{-i a \pi(t)}=\exp \left(-e^{i a \pi(t)} Q e^{-i a \pi(t)}\right)=e^{-Q-a} .
$$


Taking logarithms gives

$$
e^{i a \pi(t)} Q e^{-i a \pi(t)}=Q+a
$$

and consequently

$$
e^{i a \pi(t)} e^{i b Q} e^{-i a \pi(t)}=e^{i a b} e^{i b Q} .
$$

This is easily recognized as the Weyl form of the canonical commutation relations. Applying the Stone-von Neumann theorem [15] we conclude that $\pi=\pi_{+}$. Similarly one shows that $\pi=\pi_{-}$if $H=H_{-}$, and the case $H=H_{0}$ yields the one-dimensional representations as asserted.

We will use the notation $W_{ \pm}$for $W_{\pi_{ \pm}}$. From the explicit form (4) of the action of the Heisenberg group in an irreducible representation one obtains the action of $G$ in the representations $\pi_{ \pm}$. Using $S(a, b)=S(0, b) S(a, 0)$ the result is

$$
\pi_{ \pm}(S(a, b)) \psi(s)=e^{ \pm i b e^{-s}} \psi(s+a), \quad \psi \in \mathrm{L}^{2}(\mathbb{R}) .
$$

It is now straight-forward to determine the explicit form of $W_{ \pm}(f)$ for arbitrary $f \in \mathrm{L}^{1}\left(\mathbb{R}^{2}\right) \cap \mathscr{F}^{-1}\left(\mathrm{~L}^{1}\left(\mathbb{R}^{2}\right)\right)$. If $\langle\varphi, \psi\rangle$ denotes the inner product of $\varphi, \psi \in \mathrm{L}^{2}\left(\mathbb{R}^{2}\right)$ we get

$$
\begin{aligned}
\left\langle\varphi, W_{ \pm}(f) \psi\right\rangle & =\int d a d b d s \tilde{F} f(a, b) \bar{\varphi}(s) e^{ \pm i b e^{-s}} \psi(s+a) \\
& =\int d s d u d b \bar{\varphi}(s) \mathcal{F} f(u-s, b) e^{ \pm i b e^{-s}} \psi(u) \\
& =\sqrt{2 \pi} \int d s d u \bar{\varphi}(s) \tilde{f}\left(u-s, \pm e^{-s}\right) \psi(u) .
\end{aligned}
$$

Hence we have shown

Proposition 2.6. For $f \in \mathrm{L}^{1}\left(\mathbb{R}^{2}\right) \cap \mathcal{F}^{-1}\left(\mathrm{~L}^{1}\left(\mathbb{R}^{2}\right)\right)$ the operators $W_{ \pm}(f)$ are integral operators on $\mathrm{L}^{2}(\mathbb{R})$ with kernels given by

$$
K_{f}^{ \pm}(s, u)=\sqrt{2 \pi} \tilde{f}\left(u-s, \pm e^{-s}\right)=\int d v f\left(v, \pm e^{-s}\right) e^{-i v(u-s)} .
$$

As a consequence we can establish the following basic identities.

Proposition 2.7. (a) $W_{ \pm}(f)$ is of Hilbert-Schmidt type if and only if the restriction of $f$ to $\mathbb{R} \times \mathbb{R}_{ \pm}$is square integrable with respect to the measure

$$
d \mu=|\beta|^{-1} d \alpha d \beta
$$

and we have

$$
\left\|W_{ \pm}(f)\right\|_{2}^{2}=2 \pi \int_{\mathbb{R}} d \alpha \int_{\mathbb{R}_{ \pm}} \frac{d \beta}{|\beta|}|f(\alpha, \beta)|^{2}=2 \pi \int_{\mathbb{R}^{2}} d s d v\left|f\left(v, \pm e^{-s}\right)\right|^{2}
$$


where $\|\cdot\|_{2}$ denotes the Hilbert-Schmidt norm.

(b) If $W_{ \pm}(f)$ is trace class then

$$
\operatorname{tr} W_{ \pm}(f)=\int_{\mathbb{R}^{2}} d s d v f\left(v, \pm e^{-s}\right) .
$$

Proof. (a) The operator $W_{ \pm}(f)$ is Hilbert-Schmidt if and only if its kernel is square integrable. From Proposition 2.6 we get

$$
\int d s d u\left|K_{f}^{ \pm}(s, u)\right|^{2}=2 \pi \int d s d u\left|\tilde{f}\left(u-s, \pm e^{-s}\right)\right|^{2}=2 \pi \int d s d u\left|\tilde{f}\left(u, \pm e^{-s}\right)\right|^{2} .
$$

Applying the Plancherel theorem on the $u$-integral then proves the first assertion as well as (24).

(b) If $W_{ \pm}(f)$ is trace class, then

$$
\operatorname{tr} W_{ \pm}(f)=\int_{\mathbb{R}^{2}} d s K_{f}^{ \pm}(s, s),
$$

and (25) follows from Proposition 2.6.

We note that although $\mathscr{B}$ is not contained in $\mathrm{L}^{2}\left(\mathbb{R}^{2}, d \mu\right)$ we have that $\mathscr{B} \cap$ $\mathrm{L}^{2}\left(\mathbb{R}^{2}, d \mu\right)$ is dense in $\mathrm{L}^{2}\left(\mathbb{R}^{2}, d \mu\right)$. Indeed, let $\mathscr{B}^{\prime}$ denote the subspace of $\mathcal{B}$ consisting of Fourier transforms of the derivative with respect to the second variable of functions in $S_{\mathrm{c}}$. A function $f(\alpha, \beta)$ in $\mathscr{B}^{\prime}$ is then of the form $\beta g(\alpha, \beta)$ where $g$ is a Schwartz function, hence $f \in \mathrm{L}^{2}\left(\mathbb{R}^{2}, d \mu\right)$. Moreover, if $f$ is orthogonal to $\mathscr{B}^{\prime}$ in $\mathrm{L}^{2}\left(\mathbb{R}^{2}, d \mu\right)$ then its Fourier transform, considered as a tempered distribution, vanishes as a distribution, hence also as a tempered distribution. Thus $f=0$ and we conclude that $\mathscr{B}^{\prime}$ is dense in $\mathrm{L}^{2}\left(\mathbb{R}^{2}, d \mu\right)$.

It follows from this remark and (24) that the mappings $W_{ \pm}$have unique extensions from $\mathcal{B}^{\prime}$ to $\mathrm{L}^{2}\left(\mathbb{R}^{2}, d \mu\right)$ such that (24) still holds. In particular, the map

$$
W: f \rightarrow W_{+}(f) \oplus W_{-}(f)
$$

is injective from $\mathrm{L}^{2}\left(\mathbb{R}^{2}, d \mu\right)$ into $\mathscr{H} \oplus \mathscr{H}$, where $\mathscr{H}$ denotes the space of HilbertSchmidt operators on $\mathrm{L}^{2}(\mathbb{R})$.

On the other hand, it is clear from the proof of Proposition 2.7 that any pair of kernels $K^{ \pm}$in $\mathrm{L}^{2}\left(\mathbb{R}^{2}\right)$ originate from an $f \in \mathrm{L}^{2}\left(\mathbb{R}^{2}, d \mu\right)$, i.e., $W$ is unitary up to $\sqrt{2 \pi}$. This proves the following extension result.

Theorem 2.8. Let $\mathscr{B}^{\prime}$ and $W$ be as defined above and set $\overline{\mathcal{B}}=\mathrm{L}^{2}\left(\mathbb{R}^{2}, d \mu\right)$. Then the *-product (17) and involution (18) have unique extensions from $\mathscr{B}^{\prime}$ to $\overline{\mathcal{B}}$ such that $\overline{\mathcal{B}}$ becomes a Banach algebra and $W$ an isomorphism,

$$
W(f * g)=W(f) W(g) \quad W\left(f^{*}\right)=W(f)^{*} .
$$

If we complete the algebra $\overline{\mathcal{B}}$ in the operator norm, the resulting $C^{*}$ algebra will be the direct sum of two copies of the algebra of compact operators on $L^{2}(\mathbb{R})$. 
Corollary 2.9. The integral with respect to $d \mu$ over $\mathbb{R} \times \mathbb{R}_{ \pm}$is a positive trace on $\overline{\mathcal{B}}$ in the following sense: for any $f, g \in \bar{B}$,

$$
\int d u d s\left(f * f^{*}\right)\left(u, \pm e^{-s}\right) \geq 0
$$

and

$$
\int d u d s(f * g)\left(u, \pm e^{-s}\right)=\int d u d s(g * f)\left(u, \pm e^{-s}\right) .
$$

Proof. If $f, g \in \bar{B}$ then $W(f) W(g)$ is trace class and the second identity follows from Theorem 2.8 and (25). Setting $g=f^{*}$ and using (24) gives the first inequality.

For later use we note the following identities.

Proposition 2.10. (a) If $f, g \in \bar{B}$, then

$$
\int d \alpha d \beta|\beta|^{-1}\left(f * g^{*}\right)(\alpha, \beta)=\int d \alpha d \beta|\beta|^{-1} f(\alpha, \beta) \bar{g}(\alpha, \beta) .
$$

(b) If $f, g \in \mathscr{B}$, then

$$
\begin{aligned}
\int d \alpha d \beta\left(f * g^{*}\right)(\alpha, \beta) & =\int d \alpha d \beta f(\alpha, \beta) \bar{g}(\alpha, \beta), \\
\int d \alpha d \beta f^{*}(\alpha, \beta) & =\int d \alpha d \beta \bar{f}(\alpha, \beta) .
\end{aligned}
$$

Proof. (a) Follows immediately from Proposition 2.7 and Theorem 2.8 .

(b) Using (15) and (16) as well as (12) and (13) we have

$$
\begin{aligned}
\int d \alpha d \beta\left(f * g^{*}\right)(\alpha, \beta) & =\mathscr{F}\left(f * g^{*}\right)(0) \\
& =\left(\mathcal{F}(f) \hat{*} \mathcal{F}\left(g^{*}\right)\right)(0) \\
& =\left(\mathcal{F}(f) \hat{*} \mathcal{F}(g)^{\dagger}\right)(0) \\
& =\int d a d b \mathscr{F} f\left(-a,-e^{a} b\right) \overline{\mathcal{F}} g\left(-a,-e^{a} b\right) e^{a} \\
& =\int d a d b \widetilde{F} f(a, b) \overline{\mathcal{F} g}(a, b) \\
& =\int d \alpha d \beta f(\alpha, \beta) \bar{g}(\alpha, \beta) .
\end{aligned}
$$

Similarly, we have

$$
\int d \alpha d \beta f^{*}(\alpha, \beta)=\mathscr{F}\left(f^{*}\right)(0)=\left(\mathscr{F}(f)^{\dagger}\right)(0)=\overline{\mathscr{F}(f)}(0)=\int d \alpha d \beta \bar{f}(\alpha, \beta) .
$$


In particular, it follows that

$$
\int d \alpha d \beta\left(f * f^{*}\right)(\alpha, \beta) \geq 0, \quad f \in \mathcal{B},
$$

even though in general $\int d \alpha d \beta f * g(\alpha, \beta) \neq \int d \alpha d \beta g * f(\alpha, \beta)$, i.e., $\int d \alpha d \beta$ is not a trace on $\mathcal{B}$. However, we shall see in Proposition 4.7 that $\int d \alpha d \beta$ satisfies a twisted trace property.

2.2. The left-invariant case and other star products. The above procedure can be also applied to the convolution algebra of the left invariant measure on $G$ instead of the right-invariant one. It is then convenient to use the parametrization

$$
R(a, c)=S\left(a, e^{-a} c\right), \quad a, c \in \mathbb{R},
$$

in which the left invariant measure is $d a d c$ by Lemma 2.1. Given a unitary representation $\pi$ of $G$, the corresponding quantization map $\tilde{W}_{\pi}$ is defined by

$$
\tilde{W}_{\pi}(f)=\int d a d c \mathcal{F} f(a, c) \pi(R(a, c))=\int d a d c \mathcal{F} f(a, c) \pi\left(S\left(a, e^{-a} c\right)\right)
$$

for $f \in \mathrm{L}^{1}\left(\mathbb{R}^{2}\right) \cap \mathcal{F}^{-1}\left(\mathrm{~L}^{1}\left(\mathbb{R}^{2}\right)\right)$. More generally, let us consider the map $W_{\pi}^{\varphi}$ given by

$$
W_{\pi}^{\varphi}(f)=\int d a d c \mathcal{F} f(a, c) \pi(S(a, \varphi(a) c)),
$$

where $\varphi$ is a smooth, positive function on $\mathbb{R}$. Defining for any function $f$ of two variables,

$$
(U f)(a, b)=f(a, \eta(a) b) \eta(a)
$$

where

$$
\eta(a)=\varphi(a)^{-1}
$$

through a simple change of variables in (28) we obtain

$$
W_{\pi}^{\varphi}(f)=\pi(U \mathcal{F} f) .
$$

The corresponding star product $*_{\varphi}$ and involution ${ }^{*} \varphi$ are given by

$$
f *_{\varphi} g=\frac{1}{2 \pi} \mathcal{F}^{-1} U^{-1}((U \mathcal{F} f) \hat{*}(U \mathcal{F} g))
$$

and

$$
f^{* \varphi}=\mathscr{F}^{-1} U^{-1}\left((U \mathcal{F} f)^{\dagger}\right),
$$

which are easily seen to be well defined for $f, g \in \mathscr{B}$. More explicitly, the following result holds. 
Proposition 2.11. If $f, g \in \mathcal{B}$ and $\varphi$ is a smooth, positive function on $\mathbb{R}$, then

$$
f *_{\varphi} g(\alpha, \beta)=\frac{1}{2 \pi} \int d a d b \tilde{f}\left(b, \omega(a, b) e^{a-b} \beta\right) \tilde{g}(a-b, \omega(a, a-b) \beta) e^{i \alpha a}
$$

and

$$
f^{* \varphi}(\alpha, \beta)=\frac{1}{2 \pi} \int d v \int d \alpha^{\prime} \bar{f}\left(\alpha+\alpha^{\prime}, \omega(a,-a) e^{a} \beta\right) e^{-i \alpha^{\prime} v},
$$

where

$$
\omega(a, b)=\eta(a) \varphi(b) e^{b-a} .
$$

In particular, the star product $\star$ for the left-invariant measure, obtained for $\varphi(a)=$ $e^{-a}$, becomes

$$
f \star g(\alpha, \beta)=\frac{1}{2 \pi} \int d v \int d \alpha^{\prime} f\left(\alpha, e^{v} \beta\right) g\left(\alpha+\alpha^{\prime}, \beta\right) e^{-i \alpha^{\prime} v},
$$

and the involution ${ }^{\star}$ for the left-invariant measure is

$$
f^{\star}(\alpha, \beta)=\frac{1}{2 \pi} \int d v \int d \alpha^{\prime} \bar{f}\left(\alpha+\alpha^{\prime}, e^{v} \beta\right) e^{-i \alpha^{\prime} v} .
$$

Proof. The first two identities follow by a straightforward computation using (30) and (31) and the Fourier inversion theorem. The last two identities follow from the first two after a change of variables combined with Fourier inversion. Details are left to the reader.

Definition 2.12. By $\mathscr{B}_{\varphi}$ we shall denote the involutive algebra obtained by equipping $\mathcal{B}$ with the product $*_{\varphi}$ and involution ${ }^{*} \varphi$.

Remark 2.13. In [6] a star product is obtained by a somewhat different approach involving a reducible representation of $G$ acting on functions of two variables. Although the explicit form of that star product is not given in [6], it can be verified that it indeed coincides with (17).

A family of star products that can be expressed in terms of series expansions were considered in $[12,13]$. For an appropriate class of functions the products (17) and (34) turn out to coincide with products from that class, see Proposition 4.8 below.

The star product considered in [2] (and in [3], [4], [5]) corresponds to the case $\varphi(a)=\frac{1-e^{-a}}{a}$ above and has the property that the involution equals complex conjugation. However, this property does not determine the star product uniquely among the products $*_{\varphi}$, as it holds more generally if $\varphi$ satisfies the relation

$$
\varphi(-a)=e^{a} \varphi(a), \quad a \in \mathbb{R} .
$$

The form of the Weyl operators $W_{ \pm}^{\varphi}(f)$ for $\pi=\pi_{ \pm}$is obtained from (29) and Proposition 2.6 by an easy computation that we omit. The result is the following. 
Proposition 2.14. Assume that $\varphi$ is positive and smooth. For $f \in \mathrm{L}^{1}\left(\mathbb{R}^{2}\right) \cap$ $\mathscr{F}^{-1}\left(\mathrm{~L}^{1}\left(\mathbb{R}^{2}\right)\right)$ the operators $W_{ \pm}^{\varphi}(f)$ are integral operators on $\mathrm{L}^{2}(\mathbb{R})$ with kernels given by

$$
K_{f}^{ \pm}(s, u)=\sqrt{2 \pi} \tilde{f}\left(u-s, \pm \varphi(u-s) e^{-s}\right)=\int d v f\left(v, \pm \varphi(u-s) e^{-s}\right) e^{-i v(u-s)}
$$

It can now be seen that the norm and trace formulas (24) and (25) hold independently of the choice of $\varphi$.

Proposition 2.15. (a) $W_{ \pm}^{\varphi}(f)$ is Hilbert-Schmidt if and only if the restriction of $f$ to $\mathbb{R} \times \mathbb{R}_{ \pm}$is square integrable with respect to the measure $d \mu$ and we have

$$
\left\|W_{ \pm}^{\varphi}(f)\right\|_{2}^{2}=2 \pi \int_{\mathbb{R}^{2}} d s d v\left|f\left(v, \pm e^{-s}\right)\right|^{2}
$$

(b) If $W_{ \pm}^{\varphi}(f)$ is trace class, then

$$
\operatorname{tr} W_{ \pm}^{\varphi}(f)=\int_{\mathbb{R}^{2}} d s d v f\left(v, \pm e^{-s}\right)
$$

Proof. (a) Using Proposition 2.14 we get

$$
\begin{aligned}
\left\|W_{ \pm}^{\varphi}(f)\right\|_{2}^{2} & =2 \pi \int d u d s\left|\tilde{f}\left(u-s, \pm \varphi(u-s) e^{-s}\right)\right|^{2} \\
& =\left.2 \pi \int d v d s f\left(v, \pm \varphi(v) e^{-s}\right)\right|^{2} \\
& =2 \pi \int d v d r\left|f\left(v, \pm e^{-r}\right)\right|^{2}
\end{aligned}
$$

which coincides with (24).

(b) Similarly, we have

$$
\operatorname{tr} W_{ \pm}^{\varphi}(f)=\sqrt{2 \pi} \int d s \tilde{f}\left(0, \pm \varphi(0) e^{-s}\right)=\int d v d s f\left(v, \pm e^{-s}\right),
$$

as claimed.

Definition 2.16. Denote by $\overline{\mathcal{B}}_{\varphi}$ the Banach algebra obtained by equipping $\mathrm{L}^{2}\left(\mathbb{R}^{2}, d \mu\right)$ with the product and involution defined by (32) and (33) and extended from $\mathscr{B}^{\prime}$ using (35) in the same manner as for the case $\varphi=1$ treated previously.

Theorem 2.17. The involutive algebras $\mathcal{B}_{\varphi}$ resp. $\overline{\mathcal{B}}_{\varphi}$, where $\varphi$ is positive and smooth, are isomorphic. 
Proof. For the case of $\mathscr{B}_{\varphi}$ we note that $\mathcal{F}^{-1} U \mathcal{F}$ maps $\mathscr{B}_{\varphi}$ onto $\mathscr{B}=\mathscr{B}_{\varphi=1}$ and is by construction a homomorphism (see (15), (16), (30) and (31)). The inverse map is obtained by replacing $\varphi$ by $\eta$.

The same argument applies to $\overline{\mathscr{B}}_{\varphi}$ since Theorem 2.15 shows that $\mathcal{F}^{-1} U \mathscr{F}$ is an isometry on $\mathscr{B}^{\prime}$ and therefore its extension is an isometry from $\mathcal{B}_{\varphi}$ onto $\mathscr{B}$.

Remark 2.18. The quantization maps $W_{ \pm}$were also considered in [1] and relations (24) and (25) were likewise derived.

For the particular case $\varphi(a)=\frac{e^{a}-1}{a}$, relations (35) and (36) also appear in [2].

\section{The extended algebra}

The star algebras $\mathscr{B}_{\varphi}$ or $\overline{\mathcal{B}}_{\varphi}$ defined in the previous section obviously do not contain the coordinate functions $\alpha$ and $\beta$. Hence, to obtain a representation of $M_{\kappa}$ with $\alpha$ and $\beta$ representing the generators $t$ and $x$ in (9) we need an extension of the domain of definition for the star product and involution. It is the purpose of this section to exhibit such a construction.

As originally mentioned in [10] and developed in [11], $M_{\kappa}$ has a natural structure of an involutive Hopf algebra, which arises by dualization of the momentum subalgebra of the $\kappa$-Poincaré Hopf algebra. The coalgebra structure $(\triangle, \varepsilon)$ and antipode $S$ are defined by

$$
\begin{aligned}
\triangle t & =t \otimes 1+1 \otimes t, \quad \Delta x=x \otimes 1+1 \otimes x, \\
\varepsilon(t) & =\varepsilon(x)=0, \\
S(t) & =-t, \quad S(x)=-x .
\end{aligned}
$$

It is easy to see that the natural involution

$$
t^{*}=t, \quad x^{*}=x
$$

is compatible with the Hopf algebra structure.

As will be seen later in Corollary 3.11, the extension we present allows a generalization of the full involutive Hopf algebra structure of $\kappa$-Minkowski space. Unless explicitly stated otherwise we work with the star product associated with the rightinvariant measure because of its simple form (17). Analogous results for the $\star$-product (34) are obtained similarly. By abuse of notation we shall denote all coproducts appearing below by $\triangle$ and similarly for counits and antipodes.

3.1. The algebra $\mathcal{C}$. Using standard notation $\partial_{\alpha}^{n}=\prod_{i=1}^{k} \frac{\partial^{n_{i}}}{\partial \alpha_{i} n_{i}}, \alpha=\left(\alpha_{1}, \ldots, \alpha_{k}\right) \in$ $\mathbb{R}^{k}, n=\left(n_{1}, \ldots, n_{k}\right) \in \mathbb{N}_{0}^{k}, \mathbb{N}_{0}=\{0,1,2,3, \ldots\}$, and with $|\cdot|$ denoting the Euclidean norm on $\mathbb{R}^{k}$ we introduce the following function spaces. 
Definition 3.1. Let $\ell_{k}$ be the space of smooth functions $f(\alpha, \beta)$ on $\mathbb{R}^{2 k}$ satisfying polynomial bounds of the form

$$
\left|\partial_{\alpha}^{n} \partial_{\beta}^{m} f(\alpha, \beta)\right| \leq c_{n, m}(1+|\alpha|)^{N_{n}}(1+|\beta|)^{M_{n, m}}
$$

for all $\alpha, \beta \in \mathbb{R}^{k}$ and such that the Fourier transform $\tilde{f}$ of $f$ (as a tempered distribution) with respect to $\alpha$ has compact support in the variable $v$ dual to $\alpha$. Here, $n, m \in \mathbb{N}^{k}$ are arbitrary and $N_{n}, M_{n, m}$ are constants, the former of which is independent of $m$ and $c_{n, m}$ is a positive constant.

Given $f \in \ell_{k}$ we denote by $K_{f}$ the smallest compact subset of $\mathbb{R}^{k}$ such that $\operatorname{supp}(\tilde{f}) \subseteq K_{f} \times \mathbb{R}^{k}$.

For $k=1$ we set $\ell=\ell_{1}$ and we have the canonical inclusion $\ell \otimes \ell \hookrightarrow \ell_{2}$ given by

$$
(f \otimes g)\left(\alpha_{1}, \alpha_{2}, \beta_{1}, \beta_{2}\right)=f\left(\alpha_{1}, \beta_{1}\right) g\left(\alpha_{2}, \beta_{2}\right)
$$

for $f, g \in \mathcal{C}$.

Remark 3.2. Note that $\mathcal{B} \subseteq \mathcal{C}$ and if $f \in \mathcal{C}$ and $p$ is a polynomial in $\alpha, \beta$, then $p$ and $p f$ are in $\mathcal{C}$.

In order to extend the $*$-product to $\mathcal{C}$ let $f, g \in \mathcal{C}$ and define for fixed $\alpha, \beta \in \mathbb{R}$,

$$
g_{\alpha, \beta}(v)=g\left(\alpha, e^{-v} \beta\right) e^{i \alpha v}, \quad v \in \mathbb{R} .
$$

Motivated by (19) we then set

$$
(f * g)(\alpha, \beta)=\frac{1}{\sqrt{2 \pi}} \int d v \tilde{f}(v, \beta) g_{\alpha, \beta}(v),
$$

which is well defined since $\tilde{f}(v, \beta)$ has compact support in $v$ and $g_{\alpha, \beta}$ is a smooth function. It is a consequence of Lemma 3.3 below that $f * g \in \mathcal{C}$ and that

$$
K_{f * g} \subseteq K_{f}+K_{g} .
$$

It is useful to extend the product (19), considered as a linear map on $\mathcal{E} \otimes$, to functions $F \in \mathscr{C}_{2}$. We denote this extended product by $m_{*}$ and set

$$
\begin{aligned}
\left(m_{*} F\right)(\alpha, \beta) & =\frac{1}{2 \pi} \int d \alpha^{\prime} \int d v \chi_{F}^{1}(v) F\left(\alpha^{\prime}, \alpha, \beta, e^{-v} \beta\right) e^{i\left(\alpha-\alpha^{\prime}\right) v} \\
& =\frac{1}{2 \pi} \int d \alpha^{\prime} \int d v \chi_{F}^{1}(v) F\left(\alpha+\alpha^{\prime}, \alpha, \beta, e^{-v} \beta\right) e^{-i \alpha^{\prime} v}, F \in \mathcal{C}_{2},
\end{aligned}
$$

where $\chi_{F}^{1}$ denotes a smooth function on $\mathbb{R}$ of compact support such that

$$
\chi_{F}^{1}\left(v_{1}\right) \tilde{F}\left(v_{1}, v_{2}, \beta_{1}, \beta_{2}\right)=\tilde{F}\left(v_{1}, v_{2}, \beta_{1}, \beta_{2}\right)
$$

as distributions, that is, $\chi_{F}^{1}$ equals 1 on a neighborhood of the projection of $K_{F}$ on the first axis. Note that (39) coincides with (38) if $F=f \otimes g, f, g \in \mathcal{C}$. 
Lemma 3.3. If $F \in \mathcal{C}_{2}$, then $m_{*} F \in \mathcal{C}$ and

$$
K_{m_{*} F} \subseteq\left\{v_{1}+v_{2} \mid\left(v_{1}, v_{2}\right) \in K_{F}\right\} .
$$

Proof. First, let us verify that the double integral in (39) is convergent as a consequence of the polynomial bounds (37) for $F$. Let $\zeta$ be a smooth function of compact support on $\mathbb{R}$ that equals 1 on a neighborhood of 0 and write the integral in (39) as a sum of two terms $F_{1}(\alpha, \beta)$ and $F_{2}(\alpha, \beta)$ where

$$
F_{1}(\alpha, \beta)=\frac{1}{2 \pi} \int d \alpha^{\prime} \int d v \zeta\left(\alpha-\alpha^{\prime}\right) \chi_{F}(v) F\left(\alpha^{\prime}, \alpha, \beta, e^{-v} \beta\right) e^{i\left(\alpha-\alpha^{\prime}\right) v} .
$$

Obviously, this latter integral is absolutely convergent and by repeated differentiation with respect to $\alpha, \beta$ it is seen that $F_{1}$ is smooth and satisfies polynomial bounds of the form (37). For $F_{2}$, given by formula (41) with $\zeta$ replaced by $1-\zeta$, one obtains after $N$ partial integrations with respect to $v$ the expression

$$
\begin{aligned}
F_{2}(\alpha, \beta)=\frac{i^{N}}{2 \pi} \int d \alpha^{\prime} \int d v & \left(\alpha-\alpha^{\prime}\right)^{-N}\left(1-\zeta\left(\alpha-\alpha^{\prime}\right)\right) \\
& \frac{\partial^{N}}{\partial v^{N}}\left(\chi_{F}(v) F\left(\alpha^{\prime}, \alpha, \beta, e^{-v} \beta\right)\right) e^{i\left(\alpha-\alpha^{\prime}\right) v} .
\end{aligned}
$$

Choosing $N$ large enough one obtains an absolutely convergent integral as a consequence of (37), using that $N_{n}$ is independent of $m$. Applying the same argument to derivatives of the integrand with respect to $\alpha, \beta$ it follows easily that $F_{2}$ is smooth and satisfies the bounds (37). In the Appendix it is proven that $m_{*} F$ is independent of the choice of $\chi_{F}^{1}$ with the mentioned property and that

$$
\operatorname{supp}\left(m_{*} F\right) \subseteq\left\{v_{1}+v_{2} \mid\left(v_{1}, v_{2}\right) \in K_{F}\right\} \times \mathbb{R} .
$$

Since $K_{F}$ is compact, it follows that $m_{*}(F) \in \mathcal{C}$ and that (40) holds.

More generally, we can define maps $\ell_{k+1} \rightarrow \ell_{k}$ by letting $m_{*}$ act on any two pairs of variables $\left(\alpha_{i}, \beta_{i}\right),\left(\alpha_{j}, \beta_{j}\right)$ while keeping the other variables fixed. We shall use the notation $m_{*} \otimes 1$ and $1 \otimes m_{*}$ for the maps $\ell_{3} \rightarrow \ell_{2}$ where $m_{*}$ acts on $\left(\alpha_{1}, \beta_{1}\right),\left(\alpha_{2}, \beta_{2}\right)$ and $\left(\alpha_{2}, \beta_{2}\right),\left(\alpha_{3}, \beta_{3}\right)$, respectively.

We have:

Lemma 3.4. The product $m_{*}$ is associative in the sense that

$$
m_{*}\left(m_{*} \otimes 1\right)=m_{*}\left(1 \otimes m_{*}\right) .
$$

The proof of this lemma is deferred to the Appendix.

Next, we proceed to define the involution on $\mathcal{C}$. A convenient form is obtained from (20) which, after a simple change of variables, yields

$$
\int d \alpha d \beta f^{*}(\alpha, \beta) \tilde{\phi}(\alpha, \beta)=\int d v d \beta \overline{\tilde{f}}(v, \beta) \chi_{f}(v) \phi\left(-v, e^{-v} \beta\right) e^{-v},
$$


for $\phi \in S\left(\mathbb{R}^{2}\right)$, where $\chi_{f}$ is an arbitrary smooth function of compact support that equals 1 on a neighborhood of $K_{f}$. Defining

$$
\left(R_{f} \phi\right)(v, \beta)=\chi_{f}(v) \phi\left(-v, e^{-v} \beta\right) e^{-v}
$$

for $\phi \in S\left(\mathbb{R}^{2}\right)$, it is clear that $R_{f}$ is a continuous mapping from $S\left(\mathbb{R}^{2}\right)$ into itself. Hence, it follows that (44) defines $f^{*}$ as a tempered distribution for any $f \in \mathcal{C}$.

Lemma 3.5. If $f \in \mathcal{C}$, then $f^{*} \in \mathcal{C}$ and

$$
K_{f *}=-K_{f}, \quad f^{* *}=f .
$$

Proof. Let $f \in \mathcal{C}$. In order to show that $f^{*} \in \mathcal{C}$ we first note that $f^{*}$ is, in fact, a function given by the following generalization of (18):

$$
f^{*}(\alpha, \beta)=\frac{1}{2 \pi} \int d \alpha^{\prime} \int d v \chi_{f}(-v) \bar{f}\left(\alpha+\alpha^{\prime}, e^{-v} \beta\right) e^{-i \alpha^{\prime} v} .
$$

Indeed, convergence of this double integral is a consequence of (37), which can be seen by arguments similar to those for $m_{*}$ as follows. Let again $\zeta$ be a smooth function on $\mathbb{R}$ of compact support that equals 1 on a neighborhood of 0 and write the integral in (47) as a sum of two terms $f_{1}^{*}(\alpha, \beta)$ and $f_{2}^{*}(\alpha, \beta)$, where

$$
f_{1}^{*}(\alpha, \beta)=\frac{1}{2 \pi} \int d \alpha^{\prime} \int d v \zeta\left(\alpha^{\prime}\right) \chi_{f}(-v) \bar{f}\left(\alpha+\alpha^{\prime}, e^{-v} \beta\right) e^{-i \alpha^{\prime} v} .
$$

Clearly, this latter integral is absolutely convergent and by repeated differentiation with respect to $\alpha, \beta$ it follows easily that $f_{1}^{*}$ is smooth and satisfies (37). For $f_{2}^{*}$ one obtains after $N$ partial integrations with respect to $v$ the expression

$$
f_{2}^{*}(\alpha, \beta)=\frac{(-i)^{N}}{2 \pi} \int d \alpha^{\prime} \int d v\left(1-\zeta\left(\alpha^{\prime}\right)\right) \frac{\partial^{N}}{\partial v^{N}}\left(\chi_{f}(-v) \bar{f}\left(\alpha+\alpha^{\prime}, e^{-v} \beta\right)\right) e^{-i \alpha^{\prime} v}
$$

By choosing $N$ large enough this integral is absolutely convergent by (37). Applying the same argument to arbitrary derivatives of the integrand with respect to $\alpha, \beta$ it follows easily that $f_{2}^{*}$ is smooth and satisfies (37). Having proven convergence of the integral (47), its coincidence with $f^{*}$ follows easily. Hence, we have shown that $f^{*}$ belongs to $C$ and is given by (47).

We refer to the Appendix for a proof that $f^{*}$ is independent of the choice of function $\chi_{f}$ with the asserted property.

The Fourier transform of $f^{*}$ with respect to $\alpha$ is given by

$$
\widetilde{f^{*}}(\phi)=\overline{\tilde{f}}\left(R_{f} \phi\right),
$$

from which it is clear that

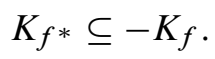


Hence we can choose $\chi_{f}^{*}(v)=\chi_{f}(-v)$. It is then easily verified that

$$
R_{f} R_{f} \phi=\chi_{f}^{2} \phi
$$

which by use of (50) gives

$$
\widetilde{f^{* *}}(\phi)=\overline{\widetilde{f}^{*}}\left(R_{f} * \phi\right)=\tilde{f}\left(R_{f} R_{f} * \phi\right)=\tilde{f}(\phi),
$$

and we know that $\chi_{f}^{2}$ equals 1 on a neighborhood of $K_{f}$. This proves the second identity in (46). Then the first relation follows from (51).

Evidently, the preceding arguments can be generalized to define an involution * on $\mathcal{C}_{k}$, for any $k \in \mathbb{N}$, by setting

$$
\begin{gathered}
F^{*}(\alpha, \beta)=(2 \pi)^{-k} \int d^{k} \alpha^{\prime} \int d^{k} v \chi_{F}(-v) \bar{F}\left(\alpha+\alpha^{\prime}, e^{-v_{1}} \beta_{1}, \ldots, e^{-v_{k}} \beta_{k}\right) \\
e^{-i\left(\alpha_{1}^{\prime} v_{1}+\cdots+\alpha_{k}^{\prime} v_{k}\right)}
\end{gathered}
$$

for $F \in \ell_{k}$, where $\chi_{F}$ is a smooth function that equals 1 on a neighborhood of $K_{F}$. Furthermore, this extension is compatible with the multiplication $m_{*}$ in the sense that

$$
\left(m_{*} F\right)^{*}=m_{*}\left(F^{*}\right)^{\wedge}, \quad F \in \mathcal{C}_{2},
$$

where ${ }^{\wedge}$ denotes the flip operation on $\mathcal{C}_{2}$,

$$
F^{\wedge}\left(\alpha_{1}, \alpha_{2}, \beta_{1}, \beta_{2}\right)=F\left(\alpha_{2}, \alpha_{1}, \beta_{2}, \beta_{1}\right) .
$$

The proof of the above statement is presented in the Appendix. As a special case we get

for all $f, g \in \mathcal{C}$.

$$
(f * g)^{*}=g^{*} * f^{*}
$$

Together with the two preceding Lemmas this implies the following result.

Proposition 3.6. $C$ equipped with the $*$-product (38) and ${ }^{*}$-involution (47) is an involutive algebra. of $\mathscr{B}$ :

This algebra can be viewed as an involutive subalgebra of the multiplier algebra

Corollary 3.7. If $f \in \mathcal{C}$ and $g \in \mathscr{B}$ then both $f * g$ and $g * f$ are in $\mathcal{B}$.

Proof. It suffices to show the result only for $f * g$, since both $\mathcal{B}$ and $\mathcal{C}$ are involutive algebras. We know that $f * g$ is in $\mathcal{C}$ so it suffices to show that it is a Schwartz function whenever $g$ is. Using (38) we have

$$
\begin{aligned}
|f * g(\alpha, \beta)|= & \frac{1}{\sqrt{2 \pi}}\left|\int d \alpha^{\prime} f\left(\alpha^{\prime}, \beta\right) \mathscr{F}\left(\chi_{f} g_{\alpha, \beta}\right)\left(\alpha^{\prime}\right)\right| \\
& \leq C(1+|\beta|)^{M}\left\|\mathcal{F}\left(\chi_{f} g_{\alpha, \beta}\right)\right\| \\
& \leq C^{\prime}(1+|\beta|)^{M}\left\|\chi_{f} g_{\alpha, \beta}\right\|^{\prime},
\end{aligned}
$$


where $\|\cdot\|,\|\cdot\|^{\prime}$ are appropriate Schwartz norms, $C, C^{\prime}$ are constants, and we have used (37). If $g$ is a Schwartz function we clearly have

$$
\left\|\chi_{f} g_{\alpha, \beta}\right\|^{\prime} \leq C_{N^{\prime}, M^{\prime}}(1+|\alpha|)^{-N^{\prime}}(1+|\beta|)^{-M^{\prime}}
$$

for arbitrary $N^{\prime}, M^{\prime} \geq 0$ and suitable constants $C_{N^{\prime}, M^{\prime}}$. Hence $f * g$ is of rapid decay. Similar arguments apply to derivatives of (38) with respect to $\alpha, \beta$, thus proving that $f * g$ is a Schwartz function if $g$ is.

Example 3.8. It is useful to note the following instances of the $*$-product.

(a) If $f, g \in \mathcal{C}$ where $g(\alpha, \beta)=g(\alpha)$ depends only on $\alpha$, then $f * g(\alpha, \beta)=$ $f(\alpha, \beta) g(\alpha)$.

(b) If $f, g \in \ell$ and $f(\alpha, \beta)=f(\beta)$ depends only on $\beta$, then $f * g(\alpha, \beta)=$ $f(\beta) g(\alpha, \beta)$.

(c) If $f(\alpha, \beta)=\alpha$ and $g \in \ell$ depends only on $\beta$, then

$$
(f * g)(\alpha, \beta)=\alpha g(\beta)+i \beta g^{\prime}(\beta), \quad(g * f)(\alpha, \beta)=g(\beta) \alpha .
$$

In particular, the constant function 1 is a unit of $\mathcal{C}$ and

$$
\alpha * g(\beta)-g(\beta) * \alpha=i \beta g^{\prime}(\beta) .
$$

For $g(\beta)=\beta$ this relation yields a representation of the defining relation (9) in terms of a $*$-commutator with $t, x$ corresponding to $\alpha, \beta$. Note also that $\alpha^{*}=\alpha$ and $\beta^{*}=\beta$ by (47).

3.2. Hopf algebra properties. We now proceed to discuss Hopf algebra features of $\ell$. More specifically, we first define maps $\Delta: \ell \rightarrow \ell_{2}, \varepsilon: \digamma \rightarrow \mathbb{C}$ and $S: \digamma \rightarrow \ell$ possessing properties of a coproduct, counit and antipode, respectively. However, the map $\Delta$ does not take values in the algebraic tensor product $\ell \otimes \ell$. We then show that by restricting to the subalgebra of polynomials in $\mathcal{C}$ we obtain a faithful realization of $M_{\kappa}$ as an involutive Hopf algebra. Furthermore, we exhibit a subalgebra $\mathcal{B}_{\mathrm{c}}$ on which $\Delta, \varepsilon$ and $S$ define the structure of a bornological quantum group.

We begin with the definition of the extended coproduct, $\triangle$, of the standard cocommutative form

$$
(\triangle f)\left(\alpha_{1}, \alpha_{2}, \beta_{1}, \beta_{2}\right)=f\left(\alpha_{1}+\alpha_{2}, \beta_{1}+\beta_{2}\right), \quad f \in \mathbb{C} .
$$

Clearly, the right-hand side is a smooth function of $\left(\alpha_{1}, \alpha_{2}, \beta_{1}, \beta_{2}\right)$ satisfying the polynomial bounds (37). Noting that

$$
\widetilde{\triangle f}\left(v_{1}, v_{2}, \beta_{1}, \beta_{2}\right)=\sqrt{2 \pi} \delta\left(v_{1}-v_{2}\right) \tilde{f}\left(v_{1}, \beta_{1}+\beta_{2}\right),
$$

where $\delta$ is the Dirac delta distribution, it follows that

$$
K_{\triangle f}=\left\{(v, v) \mid v \in K_{f}\right\}
$$


Hence $\triangle f \in \mathscr{C}_{2}$.

The maps $\Delta \otimes 1$ and $1 \otimes \Delta$ have natural extensions to maps from $\mathcal{C}_{2}$ to $\mathcal{C}_{3}$, for which we shall use the same notation:

$$
\begin{aligned}
& (\triangle \otimes 1) f\left(\alpha_{1}, \alpha_{2}, \alpha_{3}, \beta_{1}, \beta_{2}, \beta_{3}\right)=f\left(\alpha_{1}+\alpha_{2}, \alpha_{3}, \beta_{1}+\beta_{2}, \beta_{3}\right), \\
& (1 \otimes \triangle) f\left(\alpha_{1}, \alpha_{2}, \alpha_{3}, \beta_{1}, \beta_{2}, \beta_{3}\right)=f\left(\alpha_{1}, \alpha_{2}+\alpha_{3}, \beta_{1}, \beta_{2}+\beta_{3}\right) .
\end{aligned}
$$

The maps $\varepsilon: \mathcal{C} \rightarrow \mathbb{C}$ and $S: \mathcal{C} \rightarrow \mathcal{C}$ are defined by

$$
\varepsilon(f)=f(0,0)
$$

and

$$
(S f)(\alpha, \beta)=\bar{f}^{*}(-\alpha,-\beta),
$$

respectively, for $f \in \mathcal{C}$. Obviously, $\varepsilon$ and $S$ are linear maps.

In order to state the main properties of these maps we note that $\ell_{2}$ is an involutive algebra, containing $\mathcal{C} \otimes \mathcal{C}$ as an involutive subalgebra, with the $*$-product defined by a straightforward generalization of (38) to functions of 4 variables, that is,

$$
\begin{aligned}
& (F * G)\left(\alpha_{1}, \alpha_{2}, \beta_{1}, \beta_{2}\right) \\
& =\frac{1}{(2 \pi)^{2}} \int d v_{1} \int d v_{2} \int d \alpha_{1}^{\prime} \int d \alpha_{2}^{\prime} F\left(\alpha_{1}+\alpha_{1}^{\prime}, \alpha_{2}+\alpha_{2}^{\prime}, \beta_{1}, \beta_{2}\right) \\
& G\left(\alpha_{1}, \alpha_{2}, e^{-v_{1}} \beta_{1}, e^{-v_{2}} \beta_{2}\right) e^{-i\left(\alpha_{1}^{\prime} v_{1}+\alpha_{2}^{\prime} v_{2}\right)}
\end{aligned}
$$

for $F, G \in \mathcal{C}_{2}$. The involution on $\mathcal{C}_{2}$ has already been defined in (52).

Theorem 3.9. The maps $\triangle, \varepsilon, S$ satisfy the following properties.

(a) $\triangle: \mathcal{C} \rightarrow \mathscr{C}_{2}$ and $\varepsilon: \mathcal{C} \rightarrow \mathbb{C}$ are homomorphisms of involutive algebras.

(b) $S$ is an antihomomorphism of the involutive algebra $\mathcal{C}$ fulfilling

$$
S^{2}=\mathrm{Id} e .
$$

(c) $\triangle$ is coassociative in the sense that

$$
(\triangle \otimes 1) \triangle=(1 \otimes \triangle) \triangle
$$

as maps from $\mathcal{C}_{\text {to }} \mathcal{C}_{3}$.

(d) We have

$$
m_{*}(S \otimes 1) \triangle=1 \lessdot \varepsilon=m_{*}(1 \otimes S) \triangle,
$$

where $S \otimes 1$ and $1 \otimes S$ denote the natural extensions to $C_{2}$ of the corresponding operators on $\mathcal{C} \mathcal{C}$. 
Proof. (a) That $\varepsilon$ is an algebra homomorphism follows by inserting $\alpha=\beta=0$ in (38), which gives

$$
\varepsilon(f * g)=\frac{1}{\sqrt{2 \pi}} \int d v \tilde{f}(v, 0) g(0,0)=f(0,0) g(0,0)=\varepsilon(f) \varepsilon(g) .
$$

To show that $\triangle$ is an algebra homomorphism we write (58) as

$$
\begin{aligned}
& (\triangle f) *(\Delta g)\left(\alpha_{1}, \alpha_{2}, \beta_{1}, \beta_{2}\right) \\
& \left.\quad=\frac{1}{2 \pi} \int d v_{1} d v_{2} \widetilde{\triangle f}\left(v_{1}, v_{2}, \beta_{1}, \beta_{2}\right) \Delta g\left(\alpha_{1}, \alpha_{2}, e^{v_{1}} \beta_{1}, e^{v_{2}} \beta_{2}\right)\right) e^{i\left(\alpha_{1} v_{1}+\alpha_{2} v_{2}\right)} .
\end{aligned}
$$

Using (56) this yields

$$
\begin{aligned}
(\Delta f) & *(\Delta g)\left(\alpha_{1}, \alpha_{2}, \beta_{1}, \beta_{2}\right) \\
& =\frac{1}{\sqrt{2 \pi}} \int d v \tilde{f}\left(v, \beta_{1}+\beta_{2}\right) g\left(\alpha_{1}+\alpha_{2}, e^{-v}\left(\beta_{1}+\beta_{2}\right)\right) e^{i\left(\alpha_{1}+\alpha_{2}\right) v},
\end{aligned}
$$

which is seen to be identical to $\Delta(f * g)\left(\alpha_{1}, \alpha_{2}, \beta_{1}, \beta_{2}\right)$ by (38) and (55), as desired.

It is trivially verified that $\varepsilon\left(f^{*}\right)=\varepsilon(f)$ for $f \in \mathcal{C}$. It remains to verify that

$$
(\triangle f)^{*}=\triangle\left(f^{*}\right), \quad f \in \mathcal{C} .
$$

For this purpose let $\tau \in S\left(\mathbb{R}^{4}\right)$ and use (55) together with a simple change of variables to write the action of $\widetilde{\triangle f}$ as a distribution on $\tau$ in the form

$$
\widetilde{\triangle f}(\tau)=\int d \alpha d \alpha^{\prime} d \beta d \beta^{\prime} f(\alpha, \beta) \tilde{\tau}\left(\alpha-\alpha^{\prime}, \alpha^{\prime}, \beta-\beta^{\prime}, \beta^{\prime}\right) .
$$

Clearly, $\int d \alpha^{\prime} d \beta^{\prime} \tilde{\tau}\left(\alpha-\alpha^{\prime}, \alpha^{\prime}, \beta-\beta^{\prime}, \beta^{\prime}\right)$ is a Schwartz function of $(\alpha, \beta)$, whose Fourier transform with respect to $\alpha$ at $(v, \beta)$ equals $\int d \beta^{\prime} \tau\left(-v,-v, \beta-\beta^{\prime}, \beta^{\prime}\right)$. Thus, setting

$$
(T \tau)(v, \beta)=\int d \beta^{\prime} \tau\left(v, v, \beta-\beta^{\prime}, \beta^{\prime}\right),
$$

we have

$$
\widetilde{\triangle f}(\tau)=\tilde{f}(T \tau),
$$

and hence, by (50),

$$
\widetilde{\triangle\left(f^{*}\right)}(\tau)=\widetilde{f^{*}}(T \tau)=\overline{\tilde{f}}\left(R_{f} T \tau\right) .
$$

Using (45) we have

$$
\begin{aligned}
\chi_{f}(v)\left(R_{f} T \tau\right)(v, \beta) & =\chi_{f}(v)^{2} \int d \beta^{\prime} \tau\left(-v,-v, e^{-v} \beta-\beta^{\prime}, \beta^{\prime}\right) e^{-v} \\
& =\chi_{f}(v)^{2} \int d \beta^{\prime} \tau\left(-v,-v, e^{-v}\left(\beta-\beta^{\prime}\right), e^{-v} \beta^{\prime}\right) e^{-2 v} \\
& =T\left(R_{f} \otimes R_{f}\right) \tau(v, \beta) .
\end{aligned}
$$


Inserting this into the previous equation yields

$$
\widetilde{\triangle\left(f^{*}\right)}(\tau)=\overline{\tilde{f}}\left(T\left(R_{f} \otimes R_{f}\right) \tau\right)=\overline{\widetilde{\triangle f}}\left(\left(R_{f} \otimes R_{f}\right) \tau\right)=\widetilde{(\Delta f)^{*}}(\tau),
$$

which proves (60).

(b) That $S$ is an algebra antihomomorphism follows immediately from (54) and the definition of the $*$-product. Similarly, the fact that $S$ commutes with the involution on $\mathcal{C}$ and the idempotency of $S$ follow easily from (46) and (47).

(c) Coassociativity of $\triangle$ is evident.

(d) In order to prove (59) we first use (47) and (55) to write

$$
\begin{aligned}
(S \otimes 1) & \triangle f\left(\alpha_{1}, \alpha_{2}, \beta_{1}, \beta_{2}\right) \\
& =\frac{1}{2 \pi} \int d \alpha^{\prime} \int d v^{\prime} \chi_{f}\left(v^{\prime}\right) f\left(\alpha^{\prime},-e^{v^{\prime}} \beta_{1}+\beta_{2}\right) e^{i\left(\alpha_{2}-\alpha_{1}-\alpha^{\prime}\right) v^{\prime}} .
\end{aligned}
$$

Since this expression depends on $\left(\alpha_{1}, \alpha_{2}\right)$ only through $\alpha_{1}-\alpha_{2}$ it follows immediately from (39) that $m_{*}(S \otimes 1) \triangle f$ is independent of $\alpha$. Hence we may set $\alpha=0$ and obtain

$$
\begin{aligned}
m_{*}(S \otimes 1) \triangle f(\alpha, \beta)= & \frac{1}{(2 \pi)^{2}} \int d \alpha^{\prime \prime} \int d v^{\prime \prime} \int d \alpha^{\prime} \int d v^{\prime} \chi_{f}\left(-v^{\prime \prime}\right) \\
& \chi_{f}\left(v^{\prime}\right) f\left(\alpha^{\prime},-e^{v^{\prime}} \beta+e^{-v^{\prime \prime}} \beta\right) e^{-i \alpha^{\prime \prime}\left(v^{\prime}+v^{\prime \prime}\right)} e^{-i \alpha^{\prime} v^{\prime}} .
\end{aligned}
$$

Let now $\zeta_{1}$ be a smooth function on $\mathbb{R}$ of compact support which equals 1 on a neighborhood of 0 , and define the functions $\zeta_{R}, R>0$, by $\zeta_{R}(v)=\zeta_{1}\left(\frac{v}{R}\right), v \in \mathbb{R}$. Then insert

$$
1=\left(\zeta_{R_{1}}\left(\alpha^{\prime \prime}\right)+\left(1-\zeta_{R_{1}}\left(\alpha^{\prime \prime}\right)\right)\left(\zeta_{R_{2}}\left(\alpha^{\prime}+\alpha^{\prime \prime}\right)+\left(1-\zeta_{R_{2}}\left(\alpha^{\prime}+\alpha^{\prime \prime}\right)\right)\right.\right.
$$

into the integrand and accordingly write the integral as a sum of four integrals by expanding the product on the right-hand side. By performing an adequate number of partial integrations with respect to $v^{\prime}, v^{\prime \prime}$ in the three terms containing at least one factor $\left(1-\zeta_{R_{1}}\left(\alpha^{\prime \prime}\right)\right)$ or $\left(1-\zeta_{R_{2}}\left(\alpha^{\prime}+\alpha^{\prime \prime}\right)\right)$, we obtain absolutely convergent integrals that vanish in the limit $R_{1}, R_{2} \rightarrow \infty$. In other words, the integral (61) can be obtained as the limit for $R_{1}, R_{2} \rightarrow \infty$ of the absolutely convergent integrals defined by inserting an extra convergence factor $\zeta_{R_{1}}\left(\alpha^{\prime \prime}\right) \zeta_{R_{2}}\left(\alpha^{\prime}+\alpha^{\prime \prime}\right)$. For the regularized integrals we then obtain, after integrating over $\alpha^{\prime \prime}$, the expression

$$
\begin{aligned}
& \frac{1}{(2 \pi)^{3 / 2}} \int d v^{\prime} d v^{\prime \prime} d \alpha^{\prime} \chi_{f}\left(-v^{\prime \prime}\right) \chi_{f}\left(v^{\prime}\right) R_{1} \mathcal{F}\left(\zeta_{1}\right)\left(R_{1}\left(v^{\prime}+v^{\prime \prime}\right)\right) \\
& \zeta_{1}\left(\frac{\alpha^{\prime}}{R_{2}}\right) f\left(\alpha^{\prime},-e^{v^{\prime}} \beta+e^{-v^{\prime \prime}} \beta\right) e^{-i \alpha^{\prime} v^{\prime}} .
\end{aligned}
$$

It is now easy to verify that the limit for $R_{1} \rightarrow \infty$ equals

$$
\frac{1}{2 \pi} \int d v^{\prime} d \alpha^{\prime} \chi_{f}\left(v^{\prime}\right)^{2} \zeta_{1}\left(\frac{\alpha^{\prime}}{R_{2}}\right) f\left(\alpha^{\prime}, 0\right) e^{-i \alpha^{\prime} v^{\prime}}
$$


Finally, letting $R_{2} \rightarrow \infty$ gives the result

$$
\frac{1}{\sqrt{2 \pi}} \int d \alpha^{\prime} \mathcal{F}\left(\left(\chi_{f}\right)^{2}\right)\left(\alpha^{\prime}\right) f\left(\alpha^{\prime}, 0\right)=f(0,0),
$$

where we have used that $\left(\chi_{f}\right)^{2}$ equals 1 on a neighborhood of $K_{f}$. This proves the first equality in (59). The second one follows similarly.

Remark 3.10. The demonstrated homomorphism property of $\triangle$ can be expressed more generally as

$$
M_{*}(\triangle \otimes \triangle) F=\triangle\left(m_{*} F\right), \quad F \in \bigodot_{2} .
$$

Here $M_{*}: \ell_{4} \rightarrow \ell_{2}$ denotes the canonical extension of (58) given by

$$
\begin{aligned}
M_{*} H\left(\alpha_{1}, \alpha_{2}, \beta_{1}, \beta_{2}\right)= & \frac{1}{(2 \pi)^{2}} \int d v_{1} \int d v_{2} \int d \alpha_{1}^{\prime} \int d \alpha_{2}^{\prime} \chi_{H}^{1}\left(v_{1}\right) \chi_{G}^{2}\left(v_{2}\right) \\
& H\left(\alpha_{1}+\alpha_{1}^{\prime}, \alpha_{2}+\alpha_{2}^{\prime}, \alpha_{1}, \alpha_{2}, \beta_{1}, \beta_{2}, e^{-v_{1}} \beta_{1}, e^{-v_{2}} \beta_{2}\right) \\
& e^{-i\left(\alpha_{1}^{\prime} v_{1}+\alpha_{2}^{\prime} v_{2}\right)}
\end{aligned}
$$

for $H \in \bigodot_{4}$, where $\chi_{H}^{1}, \chi_{H}^{2}$ denote smooth functions of compact support that equal 1 on a neighborhood of the projection of $K_{H}$ onto the first and second axis, respectively. The verification of (62) is left to the reader.

Corollary 3.11. Let $\mathcal{P}$ be the involutive subalgebra of $\mathcal{C}$ consisting of polynomials in $\alpha, \beta$ and define the coproduct, counit and antipode on $\mathcal{P}$ as the restrictions of $\triangle, \varepsilon$ and $S$, respectively, to $\mathcal{P}$. Then $\mathcal{P}$ is an involutive Hopf algebra isomorphic to $M_{\kappa}$.

Proof. That $\mathcal{P}$ is an involutive subalgebra of $\mathcal{C}$ generated by $\alpha$ and $\beta$ follows from the observations in Example 3.8. Indeed, it follows that the involutive algebra homomorphism $\iota: M_{\kappa} \rightarrow \ell$ defined by

$$
\iota(t)=\alpha, \quad \iota(x)=\beta,
$$

fulfills

$$
\iota(p(x, t))=p(\beta, \alpha)
$$

for any polynomial of the "normal-ordered" form $p(x, t)=\sum_{m, n} a_{n, m} x^{m} t^{n}$ in $M_{\kappa}$. Since all elements in $M_{\kappa}$ can be written in this form it follows that $\iota$ is an involutive algebra isomorphism from $M_{\kappa}$ onto $\mathcal{P}$.

Clearly, $S$ maps $\mathcal{P}$ into $\mathcal{P}$ and $\triangle$ maps $\mathcal{P}$ into $\mathcal{P} \otimes \mathcal{P}$. It then follows from Theorem 3.9 that $\mathcal{P}$ is an involutive Hopf algebra, and it remains to establish the compatibility relations

$$
\triangle \iota=\iota \otimes \iota \triangle, \quad \varepsilon \iota=\varepsilon, \quad S \iota=\iota S .
$$


Knowing that $\triangle, \varepsilon$ are homomorphisms and $S$ an antihomomorphism, it suffices to verify their validity for the generators $t, x$. For $\triangle$ and $\varepsilon$ this is obvious. As noted in Example 3.8 we have $\alpha^{*}=\alpha$ and $\beta^{*}=\beta$ such that

$$
S(\alpha)=-\alpha \quad \text { and } \quad S(\beta)=-\beta
$$

On the other hand, the antipode on $M_{\kappa}$ fulfills $S t=-t, S x=-x$, and hence the claimed relation for $S$ follows.

In order to formulate the next result we make use of an extension of the notion of a Hopf algebra in the form of a bornological Hopf algebra or a bornological quantum group ${ }^{1}$ as introduced in [19], Definition 4.4. Let $C_{\mathrm{c}}^{\infty}\left(\mathbb{R}^{2}\right)$ denote the space of smooth functions of compact support on $\mathbb{R}^{2}$. Then $\mathcal{B}_{\mathrm{c}} \equiv \mathcal{F}\left(C_{\mathrm{c}}^{\infty}\left(\mathbb{R}^{2}\right)\right)$ is a subalgebra of $\mathcal{B}$ (and $\mathscr{C}$ ). Clearly, $C_{\mathrm{c}}^{\infty}\left(\mathbb{R}^{2}\right)$ is an algebra when equipped with the convolution product (12), and the latter is bounded with respect to the von Neumann bornology consisting of subsets $X$ of functions whose support is contained in a fixed compact set and such that the set of derivatives of order at most $N$ of functions in $X$ is uniformly bounded for any $N \in \mathbb{N}$. Hence $C_{\mathrm{c}}^{\infty}\left(\mathbb{R}^{2}\right)$ is a bornological algebra. Transporting the bornology on $C_{\mathrm{c}}^{\infty}\left(\mathbb{R}^{2}\right)$ to $\mathcal{B}_{\mathrm{c}}$ by $\mathcal{F}$ we obtain that $\mathcal{B}_{\mathrm{c}}$ is a bornological algebra with respect to the $*$-product (15). It is known [14] that the multiplier algebra of $C_{\mathrm{c}}^{\infty}\left(\mathbb{R}^{2}\right)$ is the space $\mathscr{E}^{\prime}\left(\mathbb{R}^{2}\right)$ of distributions of compact support. From this we conclude that the multiplier algebra of $\mathcal{B}_{\mathrm{c}}$ equals $\mathcal{F}\left(\mathscr{E}^{\prime}\left(\mathbb{R}^{2}\right)\right)$ which is easily seen to be contained in $e$.

Corollary 3.12. The algebra $\mathcal{B}_{\mathrm{c}}$ is a bornological Hopf algebra with coproduct, counit and antipode defined as the restrictions of $\triangle, \varepsilon$ and $S$, respectively, to $\mathfrak{B}_{\mathrm{c}}$.

Proof. In the following we denote a bornological tensor products by $\hat{\otimes}$. It is well known (see e.g. [14], Section 2) that

$$
C_{\mathrm{c}}^{\infty}\left(\mathbb{R}^{2}\right) \hat{\otimes} C_{\mathrm{c}}^{\infty}\left(\mathbb{R}^{2}\right)=C_{\mathrm{c}}^{\infty}\left(\mathbb{R}^{2} \times \mathbb{R}^{2}\right) .
$$

It follows that

$$
\mathscr{B}_{\mathrm{c}} \hat{\otimes} \mathscr{B}_{\mathrm{c}}=\mathscr{B}_{\mathrm{c}}\left(\mathbb{R}^{2} \times \mathbb{R}^{2}\right),
$$

where the right-hand side denotes the space of Schwartz functions on $\mathbb{R}^{4}$ with Fourier transform of compact support, and which is a subalgebra of $\ell_{2}$. The multiplier algebra of $C_{\mathrm{c}}^{\infty}\left(\mathbb{R}^{4}\right)$ equals the space of distributions on $\mathbb{R}^{4}$ of compact support [14]. Hence the multiplier algebra of $\mathscr{B}_{\mathrm{c}} \widehat{\otimes} \mathcal{B}_{\mathrm{c}}$, denoted by $M\left(\mathscr{B}_{\mathrm{c}} \hat{\otimes} \mathscr{B}_{\mathrm{c}}\right)$, consists of distributions on $\mathbb{R}^{4}$ whose Fourier transform has compact support. From this it is evident that the restriction of $\triangle$ to $\mathscr{B}_{\mathrm{c}}$ takes values in $M\left(\mathscr{B}_{\mathrm{c}} \hat{\otimes} \mathscr{B}_{\mathrm{c}}\right)$. In view of the properties of $\triangle, \varepsilon, S$ established in Theorem 3.9 and the obvious fact that $\mathscr{B}_{\mathrm{c}}$ is invariant under $S$, it now follows easily that $\mathscr{B}_{\mathrm{c}}$ is a bornological Hopf algebra. In

\footnotetext{
${ }^{1}$ We thank Christian Voigt for suggesting this.
} 
particular, one may use the properties of the antipode to show that the left Galois maps $\gamma_{l}, \gamma_{r}: \mathscr{B}_{\mathrm{c}} \hat{\otimes} \mathscr{B}_{\mathrm{c}} \rightarrow M\left(\mathscr{B}_{\mathrm{c}} \hat{\otimes} \mathscr{B}_{\mathrm{c}}\right)$, defined by

$$
\gamma_{l}(f \otimes g)=\triangle f(g \otimes 1), \quad \gamma_{r}(f \otimes g)=\triangle f(1 \otimes g),
$$

are isomorphisms onto $\mathscr{B}_{\mathrm{c}} \hat{\otimes} \mathscr{B}_{\mathrm{c}}$, and similarly for the right Galois maps.

Remark 3.13. The previous corollary is, in fact, also a corollary of Theorem 7.5 in [19] applied to the group of affine transformations on $\mathbb{R}$. Indeed, transporting the bornological quantum group resulting from that theorem by the Fourier transformation one easily verifies that the resulting coproduct, counit and antipode coincide with those defined above. Moreover, the associated (left and right) invariant functional equals the integral with respect to the uniform measure on $\mathbb{R}^{2}$.

Remark 3.14. Above we have used the $*$-product and ${ }^{*}$-involution associated with the right-invariant Haar measure on $G$ to equip $\ell$ with a structure compatible with that of $\kappa$-Minkowski space. The reader may easily check that by similar arguments one obtains an alternative extension of $M_{\kappa}$ using the $\star$-product and ${ }^{\star}$-involution associated with the left invariant Haar measure.

\section{Lorentz covariance}

A salient feature of $\kappa$-Minkowski space is the existence of an action on it of a deformation of the universal enveloping algebra of the Poincaré Lie algebra [10], [11] called the $\kappa$-Poincaré Hopf algebra. In two dimensions, the latter is usually presented as the Hopf algebra with generators $E, P$ and $N$, the energy, momentum and Lorentz boost, respectively, fulfilling the relations

$$
\begin{aligned}
& {[P, E]=0, \quad[N, E]=P,} \\
& \triangle E=E \otimes 1+1 \otimes E, \quad \triangle P=P \otimes 1+e^{-\frac{E}{\kappa}} \otimes P, \\
& {[N, P]=\frac{\kappa}{2}\left(1-e^{-\frac{2 E}{\kappa}}\right)-\frac{1}{2 \kappa} P^{2}, \quad \triangle N=N \otimes 1+e^{-\frac{E}{\kappa}} \otimes N,}
\end{aligned}
$$

where $\kappa$ is the deformation parameter and the exponential terms are to be interpreted as formal power series in $\kappa^{-1}$. The counit annihilates the generators whereas the antipode acts according to

$$
S(E)=-E, \quad S(P)=-e^{\frac{E}{\kappa}} P, \quad S(N)=-e^{\frac{E}{\kappa}} N .
$$

As mentioned previously, the algebraic $\kappa$-Minkowski space $M_{\kappa}$ can be defined as the dual of the Hopf subalgebra generated by $E$ and $P$ [11]. It is the purpose of this section to exhibit explicitly the action of the $\kappa$-Poincaré algebra in terms of linear 
operators on $\mathcal{C}$. Moreover, we shall find that by restriction we obtain an action of the $\kappa$-Poincaré algebra on the subalgebra $\mathscr{B}$ as well.

To avoid the appearance of the exponential of $\frac{E}{\kappa}$ in (63) we prefer to introduce it as an additional invertible generator $\mathcal{E}$. Furthermore, by rescaling the generators we can eliminate $\kappa$ from the definition of the algebra (which, equivalently, amounts to putting $\kappa=1$ as in (9)). So we define the $\kappa$-Poincaré algebra $\mathcal{P}_{\kappa}$ accordingly as the Hopf algebra generated by $E, P, \mathcal{E}, N$ satisfying

$$
\begin{aligned}
& {[P, E]=[P, \mathcal{E}]=[E, \mathcal{E}]=0,} \\
& {[N, E]=P, \quad[N, \mathcal{E}]=-\mathcal{E} P, \quad[N, P]=\frac{1}{2}\left(1-\mathcal{E}^{2}\right)-\frac{1}{2} P^{2},} \\
& \triangle E=E \otimes 1+1 \otimes E, \quad \triangle P=P \otimes 1+\mathcal{E} \otimes P, \\
& \triangle \mathcal{E}=\mathcal{E} \otimes \mathcal{E}, \quad \triangle N=N \otimes 1+\mathcal{E} \otimes N,
\end{aligned}
$$

and with counit and antipode given by

$$
\begin{aligned}
& \varepsilon(E)=\varepsilon(P)=\varepsilon(N)=0, \quad \varepsilon(\mathcal{E})=1, \\
& S(E)=-E, \quad S(\mathcal{E})=\mathcal{E}^{-1}, \quad S(P)=-\mathcal{E}^{-1} P, \quad S(N)=-\mathcal{E}^{-1} N .
\end{aligned}
$$

We also observe that, although the $\kappa$-Poincaré algebra was originally introduced without involution, it is easy to verify that

$$
E^{*}=E, \quad P^{*}=P, \quad N^{*}=-N, \quad \mathcal{E}^{*}=\mathcal{E},
$$

defines an involution on $\mathcal{P}_{\kappa}$ making it an involutive Hopf algebra. Note, however, that the involution does not commute with $S$.

4.1. Action of the momentum subalgebra on $\mathcal{C}$. In order to define the action of $P, E, \mathcal{E}$ on $\mathcal{C}$ we first make a slight digression on imaginary translations of elements in $e$.

Let $f \in \mathcal{C}$. Since $\tilde{f}$ has compact support in the first variable, it follows (see e.g. [17]) that $f$ can be analytically continued to an entire function of $\alpha$. The analytic continuation will likewise be denoted by $f$ and is given by

$$
\begin{aligned}
f(\alpha+i \gamma, \beta) & =\frac{1}{\sqrt{2 \pi}} \tilde{f}\left(\chi_{f}(v) e^{i(\alpha+i \gamma) v}, \beta\right) \\
& =\frac{1}{\sqrt{2 \pi}} \int d \alpha^{\prime} f\left(\alpha+\alpha^{\prime}, \beta\right) \mathcal{F}\left(e^{-\gamma v} \chi_{f}(v)\right)\left(\alpha^{\prime}\right) .
\end{aligned}
$$

For fixed $\gamma \in \mathbb{R}$ we claim that the function $T_{\gamma} f$ defined by

$$
\left(T_{\gamma} f\right)(\alpha, \beta)=f(\alpha+i \gamma, \beta)
$$

belongs to $\mathcal{C}$. Indeed, since $\mathcal{F}\left(e^{-\gamma v} \chi_{f}(v)\right)\left(\alpha^{\prime}\right)$ is a Schwartz function of $\alpha^{\prime}$, we get immediately from (68) that the derivatives of $T_{\gamma} f$ are obtained by differentiating the 
integrand and, combining this with (37), it follows easily that $T_{\gamma} f$ fulfills polynomial bounds of the form (37). Moreover, by

$$
\widetilde{T_{\gamma} f}(v, \beta)=e^{-\gamma v} \tilde{f}(v, \beta),
$$

we have $K_{T_{\gamma} f}=K_{f}$, and hence $T_{\gamma} f \in \mathcal{C}$. Using (69) or, alternatively, the uniqueness of the analytic continuation of $f$ in $\alpha$ we conclude that the imaginary translation operators $T_{\gamma}: \mathcal{C} \rightarrow \mathcal{C}$ form a one-parameter group,

$$
T_{\gamma+\eta}=T_{\gamma} T_{\eta}, \gamma, \eta \in \mathbb{R}, \quad T_{0}=\mathrm{Id}_{e} .
$$

Similarly, $k$-parameter groups of imaginary translation operators $T_{\gamma}$ are defined on $\mathcal{C}_{k}$ for any $k \in \mathbb{N}$ and $\gamma \in \mathbb{R}^{k}$. We shall write $T_{\gamma}$ for $T_{(\gamma, \ldots, \gamma)}$, independently of $k$, for $\gamma \in \mathbb{R}$.

We next note the following two properties of these maps.

Proposition 4.1. For fixed $\gamma \in \mathbb{R}$ the map $T_{\gamma}: \mathcal{C} \rightarrow \mathcal{C}$ is an algebra automorphism, that is,

$$
T_{\gamma}\left(m_{*} F\right)=m_{*}\left(T_{\gamma} F\right), \quad F \in \mathcal{C}_{2} .
$$

Moreover,

$$
T_{\gamma}\left(f^{*}\right)=\left(T_{-\gamma} f\right)^{*}, \quad f \in \mathcal{C} .
$$

Proof. Since $T_{\gamma}^{-1}=T_{-\gamma}$, it is sufficient to verify (70) and (71). By (39) we have

$$
m_{*} T_{\gamma} F(\alpha, \beta)=\int d \alpha^{\prime} \int d v \chi_{F}^{1}(v) F\left(\alpha^{\prime}+\alpha+i \gamma, \alpha+i \gamma, \beta, e^{-v} \beta\right) e^{-i \alpha^{\prime} v} .
$$

That this is an entire function of $z=\alpha+i \gamma$ for fixed $\beta$ is seen as follows. By inserting a convergence factor $\zeta_{R}\left(\alpha^{\prime}\right)$ into the integrand we have, as seen previously, that the regularized integrals converge to the integral (72) as $R \rightarrow \infty$ for fixed $z$. It is easy to see that the convergence is uniform in $z$ on compact subsets of $\mathbb{C}$. Since the regularized integrals are obviously analytic in $z$ it follows that the same holds for (72). Hence this is the unique entire function whose restriction to $\mathbb{R}$ coincides with $m_{*} F(\alpha, \beta)$ for fixed $\beta$. But this function is by definition equal to the left-hand side of (70) for $z=\alpha+i \gamma$. This concludes the proof of (70).

Concerning (71) we note that by (47),

$$
\left(T_{-\gamma} f\right)^{*}(\alpha, \beta)=\frac{1}{2 \pi} \int d \alpha^{\prime} \int d v \chi_{f}(-v) \bar{f}\left(\alpha-i \gamma+\alpha^{\prime}, e^{-v} \beta\right) e^{-i \alpha^{\prime} v},
$$

which by similar arguments as those above is seen to be an entire function of $z=$ $\alpha+i \gamma$ for fixed $\beta$. Since it coincides with $f^{*}(\alpha, \beta)$ for $\gamma=0$ we conclude that it equals the left-hand side of (71) for all $z \in \mathbb{C}$. This proves (71). 
By the preceding analyticity argument we obtain

$$
T_{\gamma}\left(f^{*}\right)(\alpha, \beta)=\frac{1}{2 \pi} \int d \alpha^{\prime} \int d v \chi_{f}(-v) \bar{f}\left(\alpha^{\prime}, e^{-v} \beta\right) e^{-\gamma v} e^{i\left(\alpha-\alpha^{\prime}\right) v}
$$

for $f \in \mathcal{C}$, since the right-hand side is seen to be an analytic function of $z=\alpha+i \gamma$ that coincides with the right-hand side of (73) for $\gamma=0$.

Now we can state the main result of this subsection on the action of the Hopf subalgebra generated by $E, P, \mathcal{E}$, called the extended momentum algebra, on $\mathcal{C}$.

Theorem 4.2. The algebra $C$ is an involutive Hopf module algebra with respect to the following linear action of the extended momentum algebra on $\mathcal{C}$ :

$$
E \triangleright f=-i \frac{\partial f}{\partial \alpha}, \quad P \triangleright f=-i \frac{\partial f}{\partial \beta}, \quad \varepsilon \triangleright f=T_{1} f .
$$

Proof. It is clear that the actions of $E, P, \mathcal{E}$ defined by (75) are linear on $\mathcal{E}$ and are mutually commuting. Therefore, it only remains to verify the compatibility of the action with the $*$-product and involution, that is, to establish

$$
A \triangleright(f * g)=m_{*}(\triangle A) \triangleright(f \otimes g), \quad(A \triangleright f)^{*}=(S A) \triangleright f^{*},
$$

for $A=E, \mathcal{E}, P$ and $f, g \in \mathcal{C}$. We first prove the first relation in the more general case where $f \otimes g$ is replaced by $F \in \mathscr{C}$. That

$$
E \triangleright\left(m_{*} F\right)=m_{*}(E \otimes 1) \triangleright F+m_{*}(1 \otimes E) \triangleright F
$$

is obvious from (39) since differentiation with respect to $\alpha$ in the integrand is permitted by a standard convergence argument. For the action of $\mathcal{E}$ we have that

$$
\mathcal{E} \triangleright\left(m_{*} F\right)=m_{*}(\mathcal{E} \otimes \mathcal{E}) \triangleright F,
$$

which is a special case of (70). Finally, for the action of $P$ we have

$$
\begin{array}{r}
\left(P \triangleright\left(m_{*} F\right)\right)(\alpha, \beta)=\frac{-i}{(2 \pi)^{2}} \int d \alpha^{\prime} \int d v \chi_{F}^{1}(v)\left(\frac{\partial F}{\partial \beta_{1}}\left(\alpha^{\prime}, \alpha, \beta, e^{-v} \beta\right)\right. \\
\left.+e^{-v} \frac{\partial F}{\partial \beta_{2}}\left(\alpha^{\prime}, \alpha, \beta, e^{-v} \beta\right)\right) e^{i\left(\alpha-\alpha^{\prime}\right) v},
\end{array}
$$

where the contribution from the first term in parenthesis is equal to $m_{*}(P \otimes 1) F(\alpha, \beta)$.

On the other hand, from (68) we get

$$
\begin{aligned}
& ((\mathcal{E} \otimes 1) \triangleright F)\left(\alpha_{1}, \alpha_{2}, \beta_{1}, \beta_{2}\right) \\
& \quad=\frac{1}{2 \pi} \int d \alpha_{1}^{\prime} \int d v_{1} \chi_{F}^{1}\left(v_{1}\right) F\left(\alpha_{1}+\alpha_{1}^{\prime}, \alpha_{2}, \beta_{1}, \beta_{2}\right) e^{-v_{1}} e^{-i \alpha_{1}^{\prime} v_{1}}
\end{aligned}
$$


and hence

$$
\begin{aligned}
m_{*}((\& \otimes 1) \triangleright F)(\alpha, \beta)=\frac{1}{2 \pi} \int d \alpha_{2}^{\prime} \int d v_{2} \int d \alpha_{1}^{\prime} \int d v_{1} \chi_{F}^{1}\left(v_{1}\right) \chi_{F}^{1}\left(v_{2}\right) \\
F\left(\alpha+\alpha_{1}^{\prime}+\alpha_{2}^{\prime}, \alpha, \beta, e^{-v_{2}} \beta\right) e^{-v_{1}} e^{-i \alpha_{1}^{\prime} v_{1}+\alpha_{2}^{\prime} v_{2}} .
\end{aligned}
$$

By introducing convergence factors $\zeta_{R_{1}}\left(\alpha^{\prime \prime}\right) \zeta_{R_{2}}\left(\alpha^{\prime}+\alpha^{\prime \prime}\right)$ as in the proof of (59) above we obtain after integrating over $\alpha^{\prime \prime}$ and taking the limit $R_{1}, R_{2} \rightarrow \infty$ that $m_{*}((\varepsilon \otimes 1) \triangleright F)(\alpha, \beta)=\int d \alpha^{\prime} \int d v^{\prime} \chi_{F}^{1}\left(v^{\prime}\right)^{2} F\left(\alpha+\alpha^{\prime}, \alpha, \beta, e^{-v^{\prime}} \beta\right) e^{-v} e^{-i \alpha^{\prime} v^{\prime}}$.

Using that $\left(\chi_{F}^{1}\right)^{2}$ equals 1 on a neighborhood of the projection of $K_{F}$ onto the first axis we see that the second term in parenthesis in (77) yields the contribution $m_{*}(\varepsilon \otimes P) F(\alpha, \beta)$. Hence we have shown that

$$
P \triangleright\left(m_{*} F\right)=m_{*}(P \otimes 1) \triangleright F+m_{*}(\varepsilon \otimes P) \triangleright F, \quad F \in \mathcal{C}_{2},
$$

which concludes the argument that the action of the extended momentum algebra is compatible with multiplication on $e$.

Next we verify the second relation in (76). For $A=\varepsilon$ it follows directly from (71), whereas for $A=E$ it is a consequence of (47) by differentiating both sides with respect to $\alpha$.

Differentiating (47) with respect to $\beta$ and using (74) we obtain

$$
P \triangleright f^{*}=-\mathcal{E}(P \triangleright f)^{*},
$$

which gives

$$
(P \triangleright f)^{*}=-\left(\mathcal{E}^{-1} P\right) \triangleright f^{*} .
$$

Since $S(P)^{*}=\left(-\mathcal{E}^{-1} P\right)^{*}=-\mathcal{E}^{-1} P$, it follows that (76) is satisfied for $A=P$. This completes the proof of the theorem.

4.2. Action of $\boldsymbol{P}_{\boldsymbol{\kappa}}$ on $\boldsymbol{C}$. To represent the boost operator $N$ by a linear action on $\boldsymbol{\ell}$, we introduce the operators of multiplication by $\alpha$ and $\beta$ as

$$
\left(L_{\alpha} f\right)(\alpha, \beta)=\alpha f(\alpha, \beta), \quad\left(L_{\beta} f\right)(\alpha, \beta)=\beta f(\alpha, \beta)
$$

for $f \in \ell$.

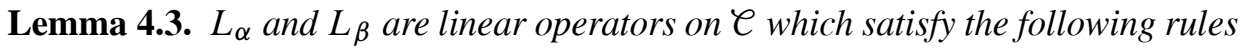
with respect to the product and involution on $\mathcal{C}$ :

$$
\begin{aligned}
L_{\alpha}\left(m_{*} F\right) & =m_{*}\left(1 \otimes L_{\alpha}\right) F=m_{*}\left(L_{\alpha} \otimes 1\right) F+m_{*}\left(1 \otimes L_{\beta} P\right) F \\
L_{\beta}\left(m_{*} F\right) & =m_{*}\left(L_{\beta} \otimes 1\right) F=m_{*}\left(\mathcal{E}^{-1} \otimes L_{\beta}\right) F \\
\left(L_{\alpha} f\right)^{*} & =L_{\alpha} f^{*}-L_{\beta} P f^{*}
\end{aligned}
$$

and

$$
\left(L_{\beta} f\right)^{*}=\varepsilon L_{\beta} f^{*}
$$


Proof. The two left identities in (79) and (80) follow immediately from (39). From the last expression in (39) we obtain

$$
\begin{aligned}
L_{\alpha}\left(m_{*} F\right)(\alpha, \beta)= & m_{*}\left(L_{\alpha} \otimes 1\right) F(\alpha, \beta) \\
& -\int d \alpha^{\prime} \int d v \chi_{F}^{1}(v) \alpha^{\prime} F\left(\alpha+\alpha^{\prime}, \alpha, \beta, e^{-v} \beta\right) e^{-i \alpha v} \\
= & m_{*}\left(L_{\alpha} \otimes 1\right) F(\alpha, \beta)+m_{*}\left(1 \otimes L_{\beta} P\right) F(\alpha, \beta),
\end{aligned}
$$

where the last step follows by a partial integration with respect to $v$. This proves the second identity in (79). Similarly, the second identity in (80) is obtained from

$$
\begin{aligned}
L_{\beta}\left(m_{*} F\right)(\alpha, \beta) & =\int d \alpha^{\prime} \int d v \chi_{F}^{1}(v) e^{v} F\left(\alpha+\alpha^{\prime}, \alpha, \beta, e^{-v} \beta\right) e^{-v} \beta e^{-i \alpha^{\prime} v} \\
& =m_{*}\left(\mathcal{E}^{-1} \otimes L_{\beta}\right) F(\alpha, \beta),
\end{aligned}
$$

where the last step follows by the same argument as in the proof of (78) above.

The identity (82) follows immediately from (47) and (74). To obtain (81) we multiply both sides of (47) by $\alpha$ and obtain after a partial integration

$$
L_{\alpha} f^{*}(\alpha, \beta)=\left(L_{\alpha} f\right)^{*}(\alpha, \beta)-\left(L_{\beta} P f\right)^{*}(\alpha, \beta) .
$$

Using (82) and the second relation in (76) for $A=P$, (81) follows.

We are now in a position to extend Theorem 4.2 as follows.

Theorem 4.4. Defining the linear action of $N$ on $\mathcal{C}$ by

$$
N=-i L_{\alpha} P-\frac{i}{2}\left(1-\varepsilon^{2}\right) L_{\beta}+\frac{i}{2} L_{\beta} P^{2}
$$

and the action of $E, P, \mathcal{E}$ as in (75), $\mathcal{C}$ becomes an involutive Hopf module algebra of $\mathcal{P}_{\kappa}$.

Proof. That $N, P, E$ and $\mathscr{E}$ satisfy the commutation relations of (64) is easily seen by inspection. It remains to check that the action of $N$ on $\mathscr{C}$ is compatible with the product and involution on $\mathcal{C}$ using the coproduct of (64). By (78) and Lemma 4.3 one gets, for $F \in \mathcal{C}_{2}$,

$$
\begin{aligned}
N \triangleright m_{*} F= & \left(-i L_{\alpha} P-\frac{i}{2}\left(1-\mathcal{E}^{2}\right) L_{\beta}+\frac{i}{2} L_{\beta} P^{2}\right) m_{*} F \\
= & -i L_{\alpha} m_{*}(P \otimes 1) F-i L_{\alpha} m_{*}(\mathcal{E} \otimes P) F \\
& -\frac{i}{2} L_{\beta} m_{*} F+\frac{i}{2} \mathcal{E}^{2} m_{*}\left(L_{\beta} \otimes 1\right) F \\
& +\frac{i}{2} L_{\beta} m_{*}\left(\left(P^{2} \otimes 1\right) F+2(\mathcal{E} P \otimes P) F+\left(\mathcal{E}^{2} \otimes P^{2}\right) F\right) .
\end{aligned}
$$


Making further use of Lemma 4.3 and (70) this expression equals

$$
\begin{aligned}
& -i m_{*}\left(L_{\alpha} P \otimes 1\right) F-i m_{*}\left(P \otimes L_{\beta} P\right) F-i m_{*}\left(\& \otimes L_{\alpha} P\right) F \\
& -\frac{i}{2} m_{*}\left(L_{\beta} \otimes 1\right) F+\frac{i}{2} m_{*}\left(\mathcal{E}^{2} L_{\beta} \otimes \mathcal{E}^{2}\right) F+\frac{i}{2} m_{*}\left(L_{\beta} P^{2} \otimes 1\right) F \\
& +i m_{*}\left(P \otimes L_{\beta} P\right) F+\frac{i}{2} m_{*}\left(\mathcal{E} \otimes L_{\beta} P^{2}\right) F .
\end{aligned}
$$

Here two terms are seen to cancel, and using the relation

$$
m_{*}\left(\mathcal{E}^{2} L_{\beta} \otimes 1\right) F-m_{*}\left(\mathcal{E} \otimes L_{\beta}\right) F=0,
$$

which follows from (80), we can rewrite the last expression in the form

$$
\begin{gathered}
m_{*}\left(-i\left(L_{\alpha} P \otimes 1\right) F-\frac{i}{2}\left(L_{\beta} \otimes 1\right) F+\frac{i}{2}\left(\mathcal{E}^{2} L_{\beta} \otimes 1\right) F+\frac{i}{2}\left(L_{\beta} P^{2} \otimes 1\right) F\right) \\
+m_{*}\left(-i\left(\mathcal{E} \otimes L_{\alpha} P\right) F-\frac{i}{2}\left(\mathcal{E} \otimes L_{\beta}\right) F+\frac{i}{2}\left(\mathcal{E} \otimes \mathcal{E}^{2} L_{\beta}\right) F+\frac{i}{2}\left(\mathcal{E} \otimes L_{\beta} P^{2}\right) F\right) \\
=m_{*}((N \otimes 1) \triangleright F+(\mathcal{E} \otimes N) \triangleright F) .
\end{gathered}
$$

This proves compatibility of the action of $N$ with the product on $\mathcal{C}$. Using the second relation in (76) for $A=P$ and $A=\&$ and (81), (82) we get

$$
\begin{aligned}
(N & \triangleright f)^{*} \\
& =\left(\left(-i L_{\alpha} P-\frac{i}{2}\left(1-\mathcal{E}^{2}\right) L_{\beta}+\frac{i}{2} L_{\beta} P^{2}\right) f\right)^{*} \\
& =\left(-i L_{\alpha} \mathcal{E}^{-1} P+i L_{\beta} P \mathcal{E}^{-1} P+\frac{i}{2}\left(1-\mathcal{E}^{-2}\right) L_{\beta} \mathcal{E}-\frac{i}{2} L_{\beta} \mathcal{E}^{-2} P^{2}\right) f^{*} \\
& =\left(-i L_{\alpha} P-\frac{i}{2}\left(1-\mathcal{E}^{2}\right) L_{\beta}+\frac{i}{2} L_{\beta} P^{2}\right) \mathcal{E}^{-1} f^{*} \\
& =N \mathcal{E}^{-1} \triangleright f^{*} .
\end{aligned}
$$

Hence $(S(N))^{*}=-\left(\mathcal{E}^{-1} N\right)^{*}=N \mathcal{E}^{-1}$ proves compatibility of the action of $N$ with involution.

In view of the obvious fact that $\frac{\partial}{\partial \alpha}, \frac{\partial}{\partial \beta}, L_{\alpha}, L_{\beta}$ and $T_{1}$ all map $\mathcal{B}$ into itself, the following is a consequence of Theorem 4.4.

Corollary 4.5. The subalgebra $\mathcal{B}$ of $\mathcal{C}$ is an involutive Hopf module algebra for $\mathcal{P}_{\kappa}$ with action defined by (75) and (83).

Remark 4.6. By inspection of (64), (65), (66) it is seen that setting

$$
\Lambda_{q}(E)=E, \quad \Lambda_{q}(P)=P, \quad \Lambda_{q}(\mathcal{E})=\mathcal{E}, \quad \Lambda_{q}(N)=N+q P
$$


defines a Hopf algebra automorphism $\Lambda_{q}$ of $\mathcal{P}_{\kappa}$ for each $q \in \mathbb{C}$. As a consequence, one obtains an involution on $\mathcal{P}_{\kappa}$ for any $q \in \mathbb{R}$ by replacing $N^{*}=-N$ in (67) with

$$
N^{*}=-N+q P \text {. }
$$

For this involution Theorem 4.4 is still valid if $N$ given by (83) is replaced with

$$
N^{\prime}=N+\frac{q}{2} P .
$$

The particular choice $q=1$ ensures that the operator $N^{\prime}$ is antisymmetric with respect to the $L^{2}$-inner product on $\mathcal{B}$, as is easily verified. More generally, it follows that the action of $h^{*}$ on $\mathscr{B}$ in this case coincides with that of the adjoint of $h$ with respect to the $L^{2}$-inner product on $\mathcal{B}$, for any $h \in \mathcal{P}_{\kappa}$, see Proposition 4.7 below.

On $\mathscr{B}$ the integral with respect to $d \alpha d \beta$ is a well defined linear form that we shall denote by $\int$. In the following proposition we collect some basic properties of $\int$ in relation to the module algebra structure on $\mathscr{B}$.

Proposition 4.7. (a) The integral with respect to the uniform measure on $\mathbb{R}^{2}$ is invariant under the action of $\mathcal{P}_{\kappa}$ on $\mathscr{B}$ defined above in the sense that, for any $h \in \mathcal{P}_{\kappa}$ and $f \in \mathscr{B}$,

$$
\int h \triangleright f=\varepsilon(h) \int f .
$$

(b) $\int$ is a left and right-invariant integral on $\mathbb{B}$ in the sense that

$$
\left(\int \otimes \mathrm{Id}\right) \triangle f=\int f=\left(\mathrm{Id} \otimes \int\right) \triangle f, \quad f \in \mathcal{B} .
$$

(c)

$$
\int S f=\int f \text { and } \int f *(S g)=\int g *(S f) .
$$

(d) For any $f, g \in \mathscr{B}$ and $h \in \mathcal{C}$ we have

$$
\int(h \triangleright f) * g^{*}=\int f *\left(h^{*} \triangleright g\right)^{*}
$$

if the involution on $\mathcal{P}_{\kappa}$ is defined by (84) for $q=1$ and the action of $E, P, \mathcal{E}, N$ on $\mathscr{B}$ is given by (75) and

$$
N \triangleright f=\left(-i L_{\alpha} P-\frac{i}{2}\left(1-\mathcal{E}^{2}\right) L_{\beta}+\frac{i}{2} P L_{\beta} P\right) f, \quad f \in \mathscr{B} .
$$

(e) For any $f, g \in \mathscr{B}$ we have

$$
\int f * g=\int(\mathcal{E} \triangleright g) * f
$$

which means that $\int$ is a twisted trace. 
Proof. (a) It suffices to verify (85) for the generators $E, P, \mathcal{E}$ and $N$. First, since both $E$ and $P$ act on $f$ as partial derivatives,

$$
\int d \alpha d \beta(P \triangleright f)(\alpha, \beta)=0=\int d \alpha d \beta(E \triangleright f)(\alpha, \beta) .
$$

For $\mathcal{E}$ we have

$$
\int d \alpha d \beta(\mathcal{E} \triangleright f)(\alpha, \beta)=\int d \alpha d \beta f(\alpha+i, \beta)=\int d \alpha d \beta f(\alpha, \beta)
$$

as a consequence of Cauchy's theorem. Finally, for the action of $N$, one uses the identities

$$
L_{\beta} P^{2}=P^{2} L_{\beta}-2 P, \quad L_{\alpha} P=P L_{\alpha},
$$

to deduce from the preceding results that

$$
\int d \alpha d \beta(N \triangleright f)(\alpha, \beta)=0 .
$$

This finishes the proof of (a).

(b) Identities (86) follow trivially from the translation invariance of the measure $d \alpha d \beta$.

(c) The first identity of (87) follows from (27) and (57):

$$
\begin{aligned}
\int d \alpha d \beta(S f)(\alpha, \beta) & =\int d \alpha d \beta(S f)(-\alpha,-\beta) \\
& =\int d \alpha d \beta \overline{f^{*}}(\alpha, \beta) \\
& =\int d \alpha d \beta f(\alpha, \beta) .
\end{aligned}
$$

The second identity follows from the former by using that $S$ is an antihomomorphism and $S^{2}=\operatorname{Id}$ on $\mathscr{B}$.

(d) By (26) we see that (88) is equivalent to the statement that the action of $h^{*} \in \mathcal{P}_{\kappa}$ on $\mathscr{B}$ as a linear operator on $\mathscr{B} \subset L^{2}\left(\mathbb{R}^{2}\right)$ equals the action of the adjoint of $h$ with respect to the standard inner product on $L^{2}\left(\mathbb{R}^{2}\right)$. That this holds for $E$ and $P$ is clear form (75). For $\&$ we have

$$
\int(\varepsilon \triangleright f) * g^{*}=\int \mathcal{E} \triangleright\left(f *\left(\mathcal{E}^{-1} \triangleright g^{*}\right)\right)=\int f *(\mathcal{E} \triangleright g)^{*}
$$

by (70), (71) and (85). This proves (88) for $h=\varepsilon$. Since $L_{\alpha}$ and $L_{\beta}$ are symmetric operators on $B \subset L^{2}\left(\mathbb{R}^{2}\right)$, one can now check by direct computation that $N$ as given by (89) is antisymmetric. 
(e) Using Cauchy's theorem and a change of variables we get from (17)

$$
\begin{aligned}
\int(\mathcal{E} \triangleright g) * f & =\frac{1}{2 \pi} \int d \alpha d \beta \int d v \int d \alpha^{\prime} g\left(\alpha+\alpha^{\prime}+i, \beta\right) f\left(\alpha, e^{-v} \beta\right) e^{-i \alpha^{\prime} v} \\
& =\frac{1}{2 \pi} \int d \alpha d \beta \int d v \int d \alpha^{\prime} g\left(\alpha^{\prime}, \beta\right) f\left(\alpha, e^{-v} \beta\right) e^{-v} e^{i\left(\alpha-\alpha^{\prime}\right) v} \\
& =\int d \beta d v \tilde{g}(v, \beta) \tilde{f}\left(-v, e^{-v} \beta\right) e^{-v} .
\end{aligned}
$$

The last expression is equal to $\int d \beta d v \tilde{f}(v, \beta) \tilde{g}\left(-v, e^{-v} \beta\right)$ as a change of variables shows, which by reversing the steps above yields $\int f * g$. This completes the proof.

4.3. Explicit dependence on the kappa parameter. For the sake of completeness we end this section by reintroducing the $\kappa$-parameter which we eliminated at the outset by rescaling the $t$ generator of $M_{\kappa}$. The correct dependence on $\kappa$ for both $M_{\kappa}$ and $\mathcal{P}_{\kappa}$ is obtained by simply rescaling the variables $\alpha, \beta$ by $\kappa$, i. e., set $(\alpha, \beta)=(\kappa \hat{\alpha}, \kappa \hat{\beta})$ and express the (co)algebra operations in terms of the dimensionful variables $\hat{\alpha}, \hat{\beta}$, and then rename the latter $(\alpha, \beta)$. Explicitly, the $*$-product on $\mathscr{B}$ is replaced by

$$
f *_{\kappa} g(\alpha, \beta)=\frac{1}{2 \pi} \int d \alpha^{\prime} d v f\left(\alpha+\alpha^{\prime}, \beta\right) g\left(\alpha, e^{-\frac{v}{\kappa}} \beta\right) e^{-i \alpha^{\prime} v},
$$

and the involution is changed to

$$
f^{*}(\alpha, \beta)=\frac{1}{2 \pi} \int d \alpha^{\prime} d v \bar{f}\left(\alpha+\alpha^{\prime}, e^{-\frac{v}{\kappa}} \beta\right) e^{-i \alpha^{\prime} v},
$$

whereas the coproduct and counit are unchanged. Furthermore, the action of the operators $E, P, \mathcal{E}, N$ on $\mathcal{B}$ are redefined by

$$
\begin{aligned}
& E \triangleright f=-i \frac{\partial f}{\partial \alpha}, \quad P \triangleright f=-i \frac{\partial f}{\partial \beta}, \quad \varepsilon \triangleright f=T_{\frac{1}{\kappa}} f, \\
& N=-i L_{\alpha} P-\frac{i \kappa}{2}\left(1-\varepsilon^{2}\right) L_{\beta}+\frac{i}{2 \kappa} L_{\beta} P^{2},
\end{aligned}
$$

where $L_{\alpha}, L_{\beta}$ denote multiplication by $\alpha, \beta$, respectively, as before. With these definitions we obtain a function algebra realization $\mathscr{B}$ of $M_{\kappa}$ and a representation of the involutive Hopf algebra $\mathcal{P}_{\kappa}$, as displayed in (63), on $\mathscr{B}$.

Finally, we note the following series representation of the $*_{\kappa}$-product, which has appeared earlier in the literature, [12], [13], for sufficiently regular functions. For simplicity we consider a rather restricted class of functions but the proof can be adapted to more general situations. 
Proposition 4.8. If $f, g \in \mathscr{B}$ and $g(\alpha, \beta)$ is an entire function of $\beta$, then

$$
\left(f *_{\kappa} g\right)(\alpha, \beta)=\sum_{n=0}^{\infty} \frac{i^{n}}{\kappa^{n} n !} \partial_{\alpha}^{n} f(\alpha, \beta)\left(\beta \partial_{\beta}\right)^{n} g(\alpha, \beta)
$$

for all $(\alpha, \beta) \in \mathbb{R}^{2}$.

Proof. First rewrite (90) as

$$
\left(f *_{\kappa} g\right)(\alpha, \beta)=\frac{1}{\sqrt{2 \pi}} \int d v \tilde{f}(v, \beta) g\left(\alpha, e^{-\frac{v}{\kappa}} \beta\right) e^{i \alpha v} .
$$

By analyticity of $g\left(\alpha, e^{-v} \beta\right)$ in $v$ we have

$$
g\left(\alpha, e^{-\frac{v}{\kappa}} \beta\right)=\sum_{n=0}^{\infty} \frac{(-1)^{n}}{\kappa^{n} n !} v^{n}\left(\beta \partial_{\beta}\right)^{n} g(\alpha, \beta) .
$$

Inserting this into the previous equation and using that the series is uniformly convergent on the compact set $K_{f}$ we get

$$
\left(f *_{\kappa} g\right)(\alpha, \beta)=\frac{1}{\sqrt{2 \pi}} \sum_{n=0}^{\infty} \frac{(-1)^{n}}{\kappa^{n} n !} \int d v \tilde{f}(v, \beta) v^{n}\left(\beta \partial_{\beta}\right)^{n} g(\alpha, \beta) e^{i \alpha v} .
$$

Now use

$$
\frac{1}{\sqrt{2 \pi}} \int \tilde{f}(v, \beta) v^{n} e^{i \alpha v}=\left(-i \partial_{\alpha}\right)^{n} f(\alpha, \beta)
$$

to conclude the proof.

\section{Conclusions}

The star product formulation of the $\kappa$-Minkowski algebra presented in this paper has potential advantages with regard to future developments. It is a basis-independent construction realized as a function space with a richer structure than the algebraic version, and with a simpler analytic form of the product than in previous approaches. We consider it as a first step towards the construction of a geometry on $\kappa$-Minkowski space in the sense of spectral triples. A primary goal will be to study the equivariant representations of the algebra $\mathcal{B}$ and to look for equivariant Dirac operators. The existence of the invariant twisted trace on $\mathscr{B}$ suggests that the geometry of $\kappa$-Minkowski space might be closer to the case of quantum groups ( $q$-deformations) than originally believed. In particular, the failure of the spectral triple construction for the compactified version of $\kappa$-Minkowski space [9] is possibly related to this fact, and the remedy might be to look for twisted spectral geometries.

Furthermore, there appear to be interesting relations between the star product formulation of $\kappa$-Minkowski space and deformations of the type considered by Rieffel [18]. We postpone the discussion of these issues, as well as extensions to higher dimensions, to a future publication. 


\section{A. Appendix}

The purpose of this appendix is to show that the definitions (39) and (47) of multiplication and inversion on $\mathcal{C}$ are independent of the choice of the functions $\chi_{f}$ and $\chi_{F}^{1}$ satisfying the stated properties and to prove (42), (43) and (53).

The support of $\boldsymbol{m}_{*} \boldsymbol{F}$. Let $F \in \mathcal{C}_{2}$. First observe that by the definition (39) of $m_{*}$ and the ensuing convergence arguments we have, for $\varphi \in S\left(\mathbb{R}^{2}\right)$,

$$
m_{*} F(\varphi)=\int d \alpha^{\prime} \int d v^{\prime} \int d \beta d \alpha \chi_{F}^{1}\left(v^{\prime}\right) F\left(\alpha+\alpha^{\prime}, \alpha, \beta, e^{-v^{\prime}} \beta\right) \varphi(\alpha, \beta) e^{-i \alpha^{\prime} v^{\prime}} .
$$

For fixed $v^{\prime}, \beta \in \mathbb{R}$ and $\xi, \eta \in S(\mathbb{R})$ we have

$$
\begin{aligned}
\int d \alpha^{\prime} d \alpha & F\left(\alpha+\alpha^{\prime}, \alpha, \beta, e^{-v^{\prime}} \beta\right) \mathcal{F} \xi\left(\alpha^{\prime}\right) \mathcal{F} \eta(\alpha) \\
= & \int d u d u^{\prime} \widetilde{F}\left(u, u^{\prime}, \beta, e^{-v^{\prime}} \beta\right) \xi(u) \eta\left(u+u^{\prime}\right) .
\end{aligned}
$$

This vanishes if $\eta\left(u+u^{\prime}\right)=0$ for all $\left(u, u^{\prime}\right) \in K_{F}$. Since this holds for arbitrary $\xi \in S(\mathbb{R})$ it follows that

$$
\int d \alpha F\left(\alpha+\alpha^{\prime}, \alpha, \beta, e^{-v^{\prime}} \beta\right) \tilde{\varphi}(\alpha, \beta)=0
$$

if $\varphi\left(u+u^{\prime}, \beta\right)=0$ for all $\left(u, u^{\prime}\right) \in K_{F}$. Hence from (A.1) we get $\widetilde{m_{*} F}(\varphi)=$ $m_{*} F(\tilde{\varphi})=0$ if $\varphi\left(u+u^{\prime}, \beta\right)$ vanishes for $\left(u, u^{\prime}\right) \in K_{F}$ for arbitrary $\beta$. This proves (42).

Independence of the $\chi$-functions. Let $f \in \mathcal{C}$ and write

$$
f^{*}=f_{1 R}^{*}+f_{2 R}^{*},
$$

where $f_{1 R}^{*}$ and $f_{2 R}^{*}$ are given by (48) and (49), respectively, with $\zeta\left(\alpha^{\prime}\right)$ replaced by $\zeta_{R}\left(\alpha^{\prime}\right)=\zeta_{1}\left(\frac{\alpha^{\prime}}{R}\right)$, and where $\zeta_{1}$ is a smooth function of compact support that equals 1 on a neighborhood of 0 . Choosing $N$ in (49) sufficiently large, it follows from (37) that $f_{2 R}^{*}$ converges to 0 uniformly on compact subsets of $\mathbb{R}^{2}$ as $R \rightarrow \infty$. Hence, $f_{1 R}^{*}$ converges uniformly to $f^{*}$ on compact subsets of $\mathbb{R}^{2}$. As the reader may easily verify, this also holds if we set $\zeta_{1}=\mathscr{F}(\zeta)$, where $\zeta$ is a smooth function with support contained in $[-1,1]$ such that $\int_{-\infty}^{\infty} \zeta(v) d v=1$, since in this case

$$
\zeta_{R}=R \mathcal{F}(\zeta(R \cdot))
$$

converges uniformly to 1 on compact subsets of $\mathbb{R}$ as $R \rightarrow \infty$. With this choice of $\zeta_{R}$ we have

$$
f_{1 R}^{*}(\alpha, \beta)=\frac{1}{\sqrt{2 \pi}} \int d v \chi_{f}(-v) \int d u R \zeta(R(v-u)) \overline{\tilde{f}}\left(-u, e^{-v} \beta\right) e^{i \alpha u} .
$$


Since the support of $u \rightarrow \zeta(R u)$ is contained in $\left[-\frac{1}{R}, \frac{1}{R}\right]$ it follows that the last integral vanishes for all $v$ outside any given distance $\delta>0$ from $-K_{f}$ if $R>\frac{1}{\delta}$. This proves that the integral defining $f^{*}$ only depends on the values of $\chi_{f}$ in any neighborhood of $K_{f}$ as desired.

The proof that $m_{*} F, F \in \mathcal{C}_{2}$, only depends on the values of $\chi_{F}^{1}$ in any neighborhood of the projection of $K_{F}$ onto the first axis is essentially identical to the preceding argument and we skip further details.

Associativity of the product. We consider $m_{*}$ given by (39) and want to verify the relation (43). For $G \in \mathscr{C}_{3}$ we have, by (39),

$$
\begin{aligned}
\left(m_{*}\right. & \otimes 1) G\left(\alpha_{1}, \alpha_{2}, \beta_{1}, \beta_{2}\right) \\
& =\frac{1}{2 \pi} \int d \alpha_{1}^{\prime} \int d v_{1} \chi_{G}^{1}\left(v_{1}\right) G\left(\alpha_{1}+\alpha_{1}^{\prime}, \alpha_{1}, \alpha_{2}, \beta_{1}, e^{-v_{1}} \beta_{1}, \beta_{2}\right) e^{-i \alpha_{1}^{\prime} v_{1}}
\end{aligned}
$$

and

$$
\begin{aligned}
m_{*}\left(m_{*} \otimes 1\right) G(\alpha, \beta) & \\
= & \frac{1}{(2 \pi)^{2}} \int d \alpha_{2}^{\prime} \int d v_{2} \int d \alpha_{1}^{\prime} \int d v_{1} \chi_{G}^{1}\left(v_{1}\right) \chi_{G}^{++}\left(v_{2}\right) \\
& \quad G\left(\alpha+\alpha_{1}^{\prime}+\alpha_{2}^{\prime}, \alpha+\alpha_{2}^{\prime}, \alpha, \beta, e^{-v_{1}} \beta, e^{-v_{2}} \beta\right) e^{-i\left(\alpha_{1}^{\prime} v_{1}+\alpha_{2}^{\prime} v_{2}\right)},
\end{aligned}
$$

where $\chi_{G}^{++}$is a smooth function of compact support that equals 1 on a neighborhood of the set $\left\{v_{1}+v_{2} \mid\left(v_{1}, v_{2}, v_{3}\right) \in K_{G}\right.$ for some $\left.v_{3} \in \mathbb{R}\right\}$. Similarly, we get

$$
\begin{aligned}
m_{*}\left(1 \otimes m_{*}\right) G(\alpha, \beta) & \\
= & \frac{1}{(2 \pi)^{2}} \int d \alpha_{1}^{\prime} \int d v_{1} \int d \alpha_{2}^{\prime} \int d v_{2} \chi_{G}^{1}\left(v_{1}\right) \chi_{G}^{2}\left(v_{2}\right) \\
& \quad G\left(\alpha+\alpha_{1}^{\prime}, \alpha+\alpha_{2}^{\prime}, \alpha, \beta, e^{-v_{1}} \beta, e^{-\left(v_{1}+v_{2}\right)} \beta\right) e^{-i\left(\alpha_{1}^{\prime} v_{1}+\alpha_{2}^{\prime} v_{2}\right)} .
\end{aligned}
$$

Now rewrite (A.2) as

$$
\begin{aligned}
m_{*}\left(m_{*} \otimes 1\right) G(\alpha, \beta) & \\
= & \frac{1}{(2 \pi)^{2}} \int d \alpha_{2}^{\prime} \int d v_{2} \int d \alpha_{1}^{\prime} \int d v_{1} \chi_{G}^{1}\left(v_{1}\right) \chi_{G}^{++}\left(v_{2}\right) \\
& \quad G\left(\alpha+\alpha_{1}^{\prime}, \alpha+\alpha_{2}^{\prime}, \alpha, \beta, e^{-v_{1}} \beta, e^{-v_{2}} \beta\right) e^{-i\left(\alpha_{1}^{\prime} v_{1}+\alpha_{2}^{\prime}\left(v_{2}-v_{1}\right)\right)}
\end{aligned}
$$

and insert convergence factors $\zeta_{R}\left(\alpha_{1}^{\prime}\right) \zeta_{R}\left(\alpha_{2}^{\prime}\right)$ to justify interchange of integrations to obtain

$$
\begin{aligned}
m_{*}\left(m_{*} \otimes 1\right) G(\alpha, \beta) & \\
= & \frac{1}{(2 \pi)^{2}} \int d \alpha_{1}^{\prime} \int d v_{1} \int d \alpha_{2}^{\prime} \int d v_{2} \chi_{G}^{1}\left(v_{1}\right) \chi_{G}^{++}\left(v_{1}+v_{2}\right) \\
& \quad G\left(\alpha+\alpha_{1}^{\prime}, \alpha+\alpha_{2}^{\prime}, \alpha, \beta, e^{-v_{1}} \beta, e^{-\left(v_{1}+v_{2}\right)} \beta\right) e^{-i\left(\alpha_{1}^{\prime} v_{1}+\alpha_{2}^{\prime} v_{2}\right)} .
\end{aligned}
$$


By an argument similar to the one proving the independence of $f^{*}$ of the choice of $\chi_{f}$ above, we may in this integral replace the function $\chi_{G}^{1}\left(v_{1}\right) \chi_{G}^{++}\left(v_{1}+v_{2}\right)$ by any smooth function of compact support that equals 1 on a neighborhood of the set $\left\{\left(v_{1}, v_{2}\right) \mid\left(v_{1}, v_{2}, v_{3}\right) \in K_{G}\right.$ for some $\left.v_{3} \in \mathbb{R}\right\}$. Since this holds for the function $\chi_{G}^{1}\left(v_{1}\right) \chi_{G}^{2}\left(v_{2}\right)$, we conclude that the integrals (A.2) and (A.3) are equal, as desired.

The *operation is an antihomomorphism. Let $F \in \ell_{2}$ and let $\chi_{F}^{+}, \chi_{F}^{1}, \chi_{F}^{2}$ denote smooth functions of compact support that equal 1 on $K_{m_{*} F}$ and the projections of $K_{F}$ onto the first and second coordinate axis, respectively. Using definitions (39) and (47) we then have

$$
\begin{aligned}
\left(m_{*} F\right)^{*}(\alpha, \beta) & \\
= & \frac{1}{(2 \pi)^{2}} \int d \alpha_{2}^{\prime} \int d v_{2} \int d \alpha_{1}^{\prime} \int d v_{1} \chi_{F}^{1}\left(v_{1}\right) \chi_{F}^{+}\left(-v_{2}\right) \\
& \quad \bar{F}\left(\alpha+\alpha_{1}^{\prime}, \alpha+\alpha_{2}^{\prime}, e^{-v_{2}} \beta, e^{-\left(v_{1}+v_{2}\right)} \beta\right) e^{i \alpha_{1}^{\prime} v_{1}-i \alpha_{2}^{\prime}\left(v_{1}+v_{2}\right)} .
\end{aligned}
$$

For the right-hand side of (53), on the other hand, we get

$$
\begin{gathered}
m_{*}\left(\left(F^{*}\right)^{\wedge}\right)(\alpha, \beta) \\
=\frac{1}{(2 \pi)^{3}} \int d \alpha^{\prime} \int d v \int d \alpha_{2}^{\prime} \int d v_{2} \int d \alpha_{1}^{\prime} \int d v_{1} \chi_{F}^{2}(-v) \chi_{F}^{2}\left(-v_{2}\right) \chi_{F}^{1}\left(-v_{1}\right) \\
\quad \bar{F}\left(\alpha+\alpha_{1}^{\prime}, \alpha_{2}^{\prime}, e^{-\left(v_{1}+v\right)} \beta, e^{-v_{2}} \beta\right) e^{-i\left(\alpha_{1}^{\prime} v_{1}+\alpha_{2}^{\prime} v_{2}\right)+i \alpha v_{2}} e^{i \alpha^{\prime}\left(v_{2}-v\right)} .
\end{gathered}
$$

Inserting convergence factors $\zeta_{R_{1}}\left(\alpha_{1}^{\prime}\right) \zeta_{R_{2}}\left(\alpha_{2}^{\prime}\right) \zeta_{R}\left(\alpha^{\prime}\right)$ into the last integral we recover its value in the limit $R, R_{1}, R_{2} \rightarrow \infty$ by the same arguments as above. By performing the $\alpha^{\prime}$-integration first in the regularized integral, we obtain

$$
\begin{aligned}
& \frac{1}{(2 \pi)^{5 / 2}} \int d v \int d \alpha_{2}^{\prime} \int d v_{2} \int d \alpha_{1}^{\prime} \int d v_{1} \chi_{F}^{2}(-v) \chi_{F}^{2}\left(-v_{2}\right) \chi_{F}^{1}\left(-v_{1}\right) \zeta_{R_{2}}\left(\alpha_{2}^{\prime}\right) \\
& \zeta_{R_{1}}\left(\alpha_{1}^{\prime}\right) \bar{F}\left(\alpha+\alpha_{1}^{\prime}, \alpha_{2}^{\prime}, e^{-\left(v_{1}+v\right)} \beta, e^{-v} \beta\right) e^{-i\left(\alpha_{1}^{\prime} v_{1}+\alpha_{2}^{\prime} v_{2}\right)+i \alpha v} \mathcal{F}\left(\zeta_{R}\right)\left(v-v_{2}\right) .
\end{aligned}
$$

In the limit $R \rightarrow \infty$ this gives

$$
\begin{aligned}
& \frac{1}{(2 \pi)^{2}} \int d \alpha_{2}^{\prime} \int d v_{2} \int d \alpha_{1}^{\prime} \int d v_{1} \chi_{F}^{2}\left(-v_{2}\right)^{2} \chi_{F}^{1}\left(-v_{1}\right) \zeta_{R_{2}}\left(\alpha_{2}^{\prime}\right) \\
& \zeta_{R_{1}}\left(\alpha_{1}^{\prime}\right) \bar{F}\left(\alpha+\alpha_{1}^{\prime}, \alpha_{2}^{\prime}, e^{-\left(v_{1}+v_{2}\right)} \beta, e^{-v_{2}} \beta\right) e^{-i\left(\alpha_{1}^{\prime} v_{1}+\alpha_{2}^{\prime} v_{2}\right)+i \alpha v_{2}} .
\end{aligned}
$$

A simple change of variables now yields

$$
\begin{aligned}
m_{*} & \left(\left(F^{*}\right)^{\wedge}\right)(\alpha, \beta) \\
= & \lim _{R_{1}, R_{2} \rightarrow \infty} \frac{1}{(2 \pi)^{2}} \int d \alpha_{2}^{\prime} \int d v_{2} \int d \alpha_{1}^{\prime} \int d v_{1} \chi_{F}^{2}\left(-v_{1}-v_{2}\right)^{2} \chi_{F}^{1}\left(v_{1}\right) \\
& \quad \zeta_{R_{2}}\left(\alpha_{2}^{\prime}\right) \zeta_{R_{1}}\left(\alpha_{1}^{\prime}\right) \bar{F}\left(\alpha+\alpha_{1}^{\prime}, \alpha+\alpha_{2}^{\prime}, e^{-v_{2}} \beta, e^{-\left(v_{1}+v_{2}\right)} \beta\right) e^{i \alpha_{1}^{\prime} v_{1}-i \alpha_{2}^{\prime}\left(v_{1}+v_{2}\right)} .
\end{aligned}
$$


Repeating previous arguments we see by choosing $\zeta_{R}$ such that $\mathcal{F}\left(\zeta_{R}\right)$ has support in $\left[\frac{1}{R}, \frac{1}{R}\right]$ that in the limit above the function $\chi_{F}^{2}\left(-v_{1}-v_{2}\right)^{2} \chi_{F}^{1}\left(v_{1}\right)$ can be replaced by any smooth function of compact support that equals 1 on a neighborhood of the set $\left\{\left(v_{1}, v_{2}\right) \mid\left(v_{1},-v_{1}-v_{2}\right) \in K_{F}\right\}$ without changing the value of the limit. Since this holds, in particular, for the function $\chi_{F}^{1}\left(v_{1}\right) \chi_{F}^{+}\left(-v_{2}\right)$, we conclude that the limit is equal to (A.4). This proves (53).

Acknowledgment. This work was supported in part by a Marie Curie Transfer of Knowledge project MTKD-CT-42360 as well as the Polish Government grant 1261/7.PRUE/2009/7.

\section{References}

[1] A. Agostini, $\kappa$-Minkowski representations on Hilbert spaces. J. Math. Phys. 48 (2007), 052305. Zbl 1144.81303 MR 2329844

[2] L. Dạbrowski and G. Piacitelli, Poincaré covariance and $\kappa$-Minkowski spacetime. Phys. Lett. A 375 (2011), 3496-3498. Zbl 1252.81077 MR 2831808

[3] L. Dạbrowski and G.Piacitelli, The $\kappa$-Minkowski spacetime: Trace, classical limit and uncertainty relations. in: The Planck scale, Proceedings of the XXV Max Born Symposium (Wroclaw, Poland, 29 June - 3 July 2009) AIP Conference Proceedings 1196, American Inst. of Physics, Melville, NY, 2009.

[4] L. Dạbrowski and G.Piacitelli, Canonical $\kappa$-Minkowski spacetime. Preprint 2010. arXiv:1004.5091

[5] L. Dạbrowski, M. Godliński, and G. Piacitelli, Lorentz covariant $\kappa$-Minkowski spacetime. Phys. Rev. D 81 (2010), 125024. MR 2749408

[6] F. D'Andrea, Spectral geometry of $\kappa$-Minkowski space. J. Math. Phys. 47 (2006), 062105. Zbl 1112.58009 MR 2239952

[7] V. Gayral, J. M. Gracia-Bondía, B. Iochum, T. Schücker, and J. C. Várilly, Moyal planes are spectral triples. Comm. Math. Phys. 246 (2004), 569-623. Zbl 1084.58008 MR 2053945

[8] I. M. Gelfand and M. A. Najmark, Unitary transformations of the group of linear transformations of the straight line. Dokl. Akad. Nauk SSSR 55 (1947), 567-570. Zbl 0029.00503 MR 0020559

[9] B. Iochum, T. Masson, T. Schücker, and A. Sitarz, Compact $\kappa$-deformation and spectral triples. Rep. Math. Phys. 68 (2011), 37-64. Zbl 06049922 MR 2846210

[10] J. Lukierski, H. Ruegg, A. Nowicki, and V. N. Tolstoy, $q$-deformation of Poincaré algebra. Phys. Lett. B 264 (1991), 331-338. MR 1121901

J. Lukierski, A. Nowicki and H. Ruegg, New quantum Poincaré algebra and $\kappa$-deformed field theory. Phys. Lett. B 293 (1992), 344-352. Zbl 0834.17022 MR 1188847

[11] S. Majid and H. Ruegg, Bicrossproduct structure of $\kappa$-Poincaré group and noncommutative geometry. Phys. Lett. B 334 (1994), 348-354.Zbl 1112.81328 MR 1290081 
[12] S. Meljanac and M. Stojić, New realizations of Lie algebra kappa-deformed Euclidean space. Eur. Phys. J. C Part. Fields 47 (2006), 531-539. Zbl 1191.81138 MR 2242642

[13] S. Meljanac and A. Samsarov, Scalar field theory on $\kappa$-Minkowski space-time and translation and Lorentz invariance. Internat. J. Modern Phys. A 26 (2011), 1439-1468. Zbl 1214.81285 MR 2786845

[14] R. Meyer, Smooth group representations on bornological vector spaces. Bull. Sci. Math. 128 (2004), 127-166. Zbl 1037.22011 MR 2039113

[15] J. v. Neumann, Die Eindeutigkeit der Schrödingerschen Operatoren. Math. Ann. 104 (1931), 570-578. JFM 57.1446.01 Zbl 0001.24703

M. H. Stone, Linear transformations in Hilbert space. III. Operational methods and group theory. Proc. Natl. Acad. Sci. USA 16 (1930), 172-175. JFM 56.0357.01

[16] G. K. Pedersen, $C^{*}$-algebras and their automorphism groups. London Math. Soc. Monogr. 14, Academic Press, London 1979. Zbl 0416.46043 MR 548006

[17] M. Reed and B. Simon, Methods of modern mathematical physics II: Fourier analysis, self-adjointness. Academic Press, New York 1975. Zbl 0308.47002 MR 0493420

[18] M. A. Rieffel, Deformation quantization for actions of $R^{d}$. Mem. Amer. Math. Soc. 106 (1993), no. 506. Zbl 0798.46053 MR 1184061

[19] C. Voigt, Bornological quantum groups. Pacific J. Math. 235 (2008), 93-135. Zbl 1157.46041 MR 2379773

Received April 30, 2011

B. Durhuus, Department of Mathematical Sciences, University of Copenhagen, Universitetsparken 5, 2100 Copenhagen, Denmark

E-mail: durhuus@math.ku.dk

A. Sitarz, Institute of Physics, Jagiellonian University, Reymonta 4, 30-059 Kraków, Poland

E-mail: sitarz@if.uj.edu.pl 